\title{
Closed-Form Expressions of Multilayered Planar Green's Functions That Account for the Continuous Spectrum in the Far Field
}

\author{
Francisco Mesa, Member, IEEE, Rafael R. Boix, Member, IEEE, and Francisco Medina, Senior Member, IEEE
}

\begin{abstract}
The rational function fitting method has been found useful in the derivation of closed-form expressions of spatial-domain Green's functions for multilayered media. However, former implementations of the rational function fitting method lead to Green's functions expressions that are not accurate in the far field when this far field is dominated by the continuous spectrum instead of being dominated by surface waves (as it happens, for instance, in the case of lossy multilayered media). In this paper, the authors introduce a novel implementation of the rational function fitting method, which leads to Green's functions expressions that are accurate in the far field when this is dominated either by the continuous spectrum or by surface waves. In the new approach, the far-field contribution of the continuous spectrum to the Green's functions is numerically fitted in terms of functions with closed-form Hankel transforms, and this far-field contribution is explicitly added to the total least squares approximations of the Green's functions. The numerical results obtained for the Green's functions with the new approach have been compared with numerical results obtained via direct numerical integration of Sommerfeld integrals, and excellent agreement has been found despite the contribution-continuous spectrum or surface waves-dominating the far field.
\end{abstract}

Index Terms-Green's functions, layered media, Sommerfeld integrals.

\section{INTRODUCTION}

$\mathbf{T}$ HE application of the method of moments to the solution of mixed potential integral equations has proven to be an efficient numerical tool for the analysis of planar circuits and antennas [1]-[4]. In fact, several commercial software products that are currently used in the design of planar structures (such as Ansoft Designer, Zeland IE3D, and Agilent Momentum) are based on the solution of mixed potential integral equations by means of the method of moments. In order to solve the mixed potential integral equations arising from the analysis of planar structures, it is necessary to calculate the spatial-domain Green's functions for the scalar and vector potentials in

Manuscript received June 20, 2007; revised April 14, 2008. First published June 20, 2008; last published July 9, 2008 (projected). This work was supported by the Spanish Ministerio de Educación y Ciencia and European Union FEDER funds under Project TEC2007-65376, and by Junta de Andalucía under Project TIC-253.

F. Mesa is with the Microwaves Group, Department of Applied Physics 1, Escuela Técnica Superior de Ingeniería Informática, University of Seville, 41012 Seville, Spain (e-mail: mesa@us.es).

R. R. Boix and F. Medina are with the Microwaves Group, Department of Electronics and Electromagnetism, College of Physics, University of Seville, 41012 Seville, Spain (e-mail: boix@us.es; medina@us.es).

Color versions of one or more of the figures in this paper are available online at http://ieeexplore.ieee.org.

Digital Object Identifier 10.1109/TMTT.2008.925576 multilayered media [5], [6]. These Green's functions can be determined by computing infinite integrals of spectral-domain Green's functions that are commonly known as Sommerfeld integrals. Unfortunately, the highly oscillatory nature of the integrands involved makes the brute-force numerical computation of Sommerfeld integrals cumbersome and time consuming [5].

Among the many different techniques that have been proposed for speeding up the evaluation of Sommerfeld integrals (see the references in [7]), the most efficient ones are those that approximate the spectral-domain Green's functions in terms of simple functions that lead to closed-form expressions for the Sommerfeld integrals (and, therefore, the spatial-domain Green's functions). These latter techniques can be sorted in two groups. The first group uses the so-called discrete complex image method, and tries to obtain approximations of the spatial-domain Green's functions that primarily consist of spherical waves, where the amplitudes of the spherical waves are computed via Prony's method or matrix pencil method [8], [9]. The main problem with the discrete complex image method is that it may lead to closed-form expressions of the spatial-domain Green's functions that deteriorate in the far field in an unpredictable way [10]-[12]. This far-field problem can be alleviated if a surface waves term is extracted from the Green's functions before the application of the method [13], [14]. The second group of techniques for the derivation of closed-form spatial Green's functions uses the rational function fitting method. In this method, the quasi-static part of the spectral-domain Green's functions is derived in closed form and the dynamic part is approximated in a pole-residue form, which yields expressions of the spatial-domain Green's functions that primarily consist of cylindrical waves [7], [15]. The amplitudes and propagation constants of these cylindrical waves can be obtained as the solutions to an eigenvalue problem [16], [17] via an iterative vector fitting algorithm [15] or via the method of total least squares [7]. In the first papers dealing with the rational function fitting method, it was found that the closed-form expressions obtained for the spatial Green's functions produced unacceptable numerical errors in the near field [16], [15], which were due to the nonphysical singularities provided by the Hankel functions representing the cylindrical waves. The authors of this paper have recently introduced a procedure that mathematically cancels these nonphysical singularities, and thus makes it possible to obtain closed-form Green's functions that are accurate in the near field [7]. An alternative solution to the near-field problems of the rational function fitting method has been recently addressed in [18], where the rational function 
fitting method is hybridized with the discrete complex image method.

Both the discrete complex image method with surface waves extraction and the rational function fitting method seem to be suitable methods for reproducing the far-field behavior of the spatial-domain Green's functions of a multilayered substrate, as demonstrated in [7], [13], and [15]. The reason for it is that the far-field behavior is frequently dominated by cylindrical surface waves whose amplitudes and propagation constants are well predicted by both methods (e.g., see [7, Table I] and [15, Table I]). However, the far-field behavior of the spatial-domain Green's functions is not only contributed by the surface waves, but is also contributed by the so-called continuous spectrum [19], [20]. The surface waves are related to poles of the spectral-domain Green's functions located on the proper Riemann sheet, and the continuous spectrum is related to the existence of branch point singularities in the spectral-domain Green's functions [19], [20]. In lossless multilayered media, the surface waves contribution usually dominates over the continuous spectrum contribution in the far field [20] (except for the case of Green's functions without TM spectral proper poles, as will be shown in Section II), and then the methods described in [7], [13], and [15] tend to provide accurate results in the far field. However, in lossy multilayered media, the cylindrical surface waves attenuate and, after a certain distance between source and observation points, the continuous spectrum dominates the far field [20]. As a consequence of this, in this case, both the discrete complex image method with surface waves extraction [13] and the rational function fitting method [7], [15] cannot provide accurate results in the far field.

In this paper, we present an enhanced version of the rational function fitting approach reported in [7], which provides accurate Green's functions in the far field when the far field is dominated by surface waves, as well as by the continuous spectrum. In the new approach, the far-field contribution of the continuous spectrum to the Green's functions is first derived in closed form. This contribution is then numerically fitted in terms of functions that provide closed-form expressions for the continuous spectrum both in the spatial and spectral domains. Once this far-field contribution is available, its spectral version is explicitly extracted from the spectral-domain Green's functions and the method of total least squares is subsequently applied to the resulting expressions [7]. This procedure leads to closed-form expressions of the spatial-domain Green's functions that explicitly contain information about the far-field contributions of both the surface waves and the continuous spectrum. Thus, these closed-form expressions accurately reproduce the far-field behavior of the spatial-domain Green's functions in all scenarios. It should be pointed out that the expressions derived in this paper for the far-field contribution of the continuous spectrum could also be explicitly incorporated in the discrete complex image method so that this method could accurately reproduce the far field of multilayered Green's functions in those circumstances where the far-field contribution of the continuous spectrum dominates over the far-field contribution of the surface waves.

This paper is organized as follows. Section II provides all the mathematical derivations that lead to the expressions of the far-field contributions of the continuous spectrum both in the spatial and spectral domains. These mathematical derivations are presented for Sommerfeld integrals involving Hankel transforms of order 0 and order 1. In Section III, the numerical results obtained for the spatial-domain Green's functions with the approach of this paper are compared with numerical results obtained via the approach of [7] and with numerical results obtained through direct numerical integration of Sommerfeld integrals. This comparison shows that whereas the approach of [7] fails to provide accurate far-field results in the cases where the far field is dominated by the continuous spectrum, the approach of this paper provides accurate far-field results both when the far field is dominated by surface waves and when the far field is dominated by the continuous spectrum. Conclusions are summarized in Section IV.

\section{ANALYSIS}

The method developed by the authors in [7] clearly shows its efficiency in a great variety of situations. One of the key points of our proposal was the ability to derive, in a systematic way, quasi-analytic expressions for the spatial-domain multilayered Green's functions that are valid in a wide range of distances. Thus, the singular, near-field, and far-field behaviors of the different Green's functions used in the mixed-potential integral-equation were altogether well accounted for by our method. Specifically, our procedure in [7] assumed that the corresponding spectral-domain Green's functions $\tilde{G}_{n}\left(k_{\rho}, z, z^{\prime}\right)(n=0,1)$ can be approximated by means of a rational fitting procedure, or equivalently by means of the following pole-residue (pr) representation:

$$
\tilde{G}_{n}^{\mathrm{TLS}(\mathrm{pr})}\left(k_{\rho}, z, z^{\prime}\right)=\sum_{i=1}^{M} \frac{a_{n, i}\left(z, z^{\prime}\right)}{k_{\rho}{ }^{2}-\left[p_{n, i}\left(z, z^{\prime}\right)\right]^{2}}, \quad n=0,1
$$

plus an asymptotic term $\tilde{G}_{n}^{\text {as }}\left(k_{\rho}, z, z^{\prime}\right)$ to build

$$
\begin{gathered}
\tilde{G}_{n}\left(k_{\rho}, z, z^{\prime}\right) \approx \tilde{G}_{n}^{\mathrm{aS}}\left(k_{\rho}, z, z^{\prime}\right)+\tilde{G}_{n}^{\mathrm{TLS}(\mathrm{pr})}\left(k_{\rho}, z, z^{\prime}\right), \\
n=0,1
\end{gathered}
$$

where the index $n(n=0,1)$ stands for a different type of mixed-potential Green's functions, as explained in [6] and [7], $\tilde{G}_{n}^{\text {as }}\left(k_{\rho}, z, z^{\prime}\right)$ represents the behavior of $\tilde{G}_{n}\left(k_{\rho}, z, z^{\prime}\right)$ for large values of $k_{\rho}$ (thus determining the behavior of $G_{n}\left(\rho, z, z^{\prime}\right)$ in the vicinity of $\left.\rho=\sqrt{\left(x-x^{\prime}\right)^{2}+\left(y-y^{\prime}\right)^{2}}=0\right)$, and $\tilde{G}_{n}^{\mathrm{TLS}(\mathrm{pr})}\left(k_{\rho}, z, z^{\prime}\right)$ is numerically obtained via the method of total least squares [7], [21], [22]. Concerning the quasi-static part of the spectrum (namely, $\tilde{G}_{n}^{\text {as }}\left(k_{\rho}, z, z^{\prime}\right)$ ), the results presented in [7] and [15] seem to indicate that $\tilde{G}_{n}^{\text {as }}\left(k_{\rho}, z, z^{\prime}\right)$ is correctly approximated by a singular term (corresponding to the source in a homogeneous medium) plus one or two static images. Our numerical simulations have shown that when the thickness of the layers surrounding the source and field points is electrically small (i.e., roughly smaller than $0.05 \lambda_{0}$, with $\lambda_{0}$ being the free-space wavelength), additional static images should be included in $\tilde{G}_{n}^{\text {as }}\left(k_{\rho}, z, z^{\prime}\right)$ for a correct performance of (1) and (2). These static images can be either exactly obtained by using the algorithm presented in [23] or approximately obtained with very little computational burden by means of Prony's method [24], 


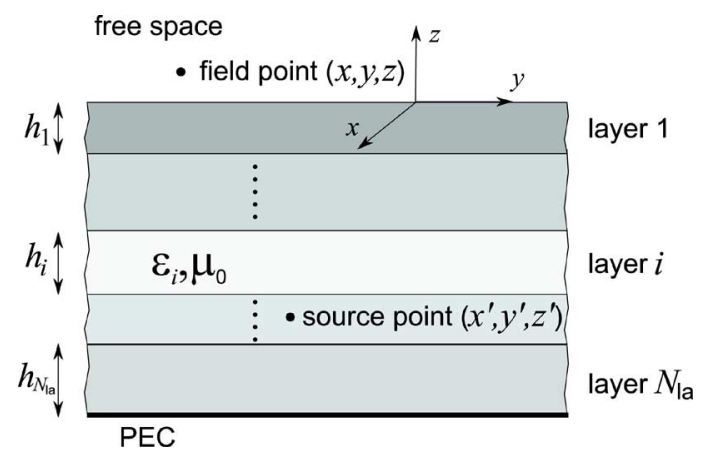

Fig. 1. Multilayered lossy medium terminated by a PEC at the bottom. The source and field points of the Green's functions can be arbitrarily placed inside the multilayered medium.

as explained in [25]. This issue was not commented upon in [7] since all the results presented in that paper were for multilayered substrates containing electrically thick layers (i.e., the thickness of the layers always happened to be larger than $0.1 \lambda_{0}$ for all the structures analyzed in that paper).

The work presented in [7] was basically aimed at developing a new procedure rather than exploiting all its capabilities in order to cover the wide range of possible physical situations that can be encountered in practice. Thus, [7] focused on the analysis of lossless structures without considering some "complex" situations that involve "anomalous" far-field behaviors [20], [26]. In this way, all the Green's functions with far-field behaviors of the type $\rho^{-1 / 2}$ were well accounted for by the convenient expansion of surface waves proposed in [7] (i.e. by a short series of Hankel functions whose complex amplitudes and wavenumbers can be computed by means of a total least squares approach). Next, our attention will especially focus on those situations not covered in [7] with the purpose of making our approach as general as possible.

\section{A. Decomposition of the Green's Function}

Fig. 1 shows a multilayered medium consisting of lossy layers of complex permittivity $\varepsilon_{i}=\varepsilon_{0} \varepsilon_{r i}\left(1-\mathrm{j} \tan \delta_{i}\right)\left(\tan \delta_{i}\right.$ will be assumed to be given by $\sigma_{i} /\left(\omega \varepsilon_{0} \varepsilon_{r i}\right)$ for layers characterized by a conductivity $\left.\sigma_{i}\right)$ and thickness $h_{i}\left(i=1, \ldots, N_{\text {la }}\right)$. The multilayered medium is assumed to be limited by free space at the upper end, and by a perfect electric conductor (PEC) at the lower end. In order to understand the appearance of far-field behaviors different from $\rho^{-1 / 2}$ in the Green's functions of the structure of Fig. 1, a convenient decomposition will be carried out in the Sommerfeld integrals that appear in the computation of those Green's functions in the spatial domain. The Sommerfeld integrals appear when performing an inverse Hankel transform to compute the spatial counterpart of the spectral-domain Green's functions [20]

$$
\begin{aligned}
& G_{n}\left(\rho, z, z^{\prime}\right) \\
& \quad=S_{n}\left[\tilde{G}_{n}\left(k_{\rho}, z, z^{\prime}\right)\right] \\
& \quad=\frac{1}{2 \pi} \int_{0(\operatorname{SIP})}^{\infty} \tilde{G}_{n}\left(k_{\rho}, z, z^{\prime}\right) J_{n}\left(k_{\rho} \rho\right) k_{\rho}{ }^{n+1} \mathrm{~d} k_{\rho} \\
& \quad=\frac{1}{4 \pi} \int_{-\infty(\operatorname{SIP})}^{\infty} \tilde{G}_{n}\left(k_{\rho}, z, z^{\prime}\right) H_{n}^{(2)}\left(k_{\rho} \rho\right) k_{\rho}{ }^{n+1} \mathrm{~d} k_{\rho}
\end{aligned}
$$

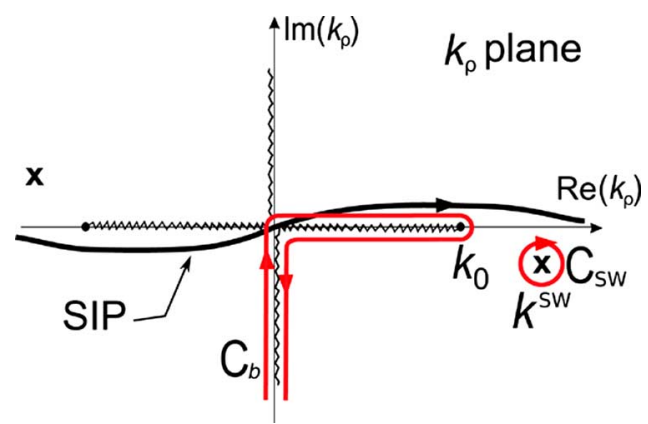

Fig. 2. Original SIP to carry out the inverse Hankel transform. The SIP is deformed to the paths $C_{b}$ and $C_{\mathrm{sw}}$. Only one surface wave pole $\left(k^{\mathrm{sw}}\right)$ is assumed to be located on the proper Riemann sheet.

where $J_{n}(\cdot)$ is the Bessel function of order $n, H_{n}^{(2)}(\cdot)$ is the Hankel function of order $n$ and second type, $\rho$ is the horizontal distance between source and field points (see Fig. 1), and the Sommerfeld integration path (SIP) in (4) is shown in Fig. 2. After deforming the original SIP to the paths shown in Fig. 2, the integral in (4) can be split into two contributions that account for the discrete and continuous spectra [19], [20], [27]-[30]

$$
G_{n}\left(\rho, z, z^{\prime}\right)=G_{n}^{\mathrm{DS}}\left(\rho, z, z^{\prime}\right)+G_{n}^{\mathrm{CS}}\left(\rho, z, z^{\prime}\right) .
$$

The discrete-spectrum contribution

$$
\begin{aligned}
& G_{n}^{\mathrm{DS}}\left(\rho, z, z^{\prime}\right) \\
& \quad=\frac{1}{4 \pi} \sum_{i=1}^{N_{\mathrm{sw}}} \int_{C_{\mathrm{Sw}}, i} \tilde{G}_{n}\left(k_{\rho}, z, z^{\prime}\right) H_{n}^{(2)}\left(k_{\rho} \rho\right) k_{\rho}{ }^{n+1} \mathrm{~d} k_{\rho} \\
& \quad=-\frac{\mathrm{j}}{2} \sum_{i=1}^{N_{\mathrm{sw}}} R_{n, i}\left(z, z^{\prime}\right) H_{n}^{(2)}\left(k_{i}^{\mathrm{sw}} \rho\right)\left(k_{i}^{\mathrm{sw}}\right)^{n+1}
\end{aligned}
$$

comprises a series of above-cutoff surface wave modes whose wavenumbers are given by the poles $k_{i}^{\mathrm{sw}}\left(i=1, \ldots, N_{\mathrm{sw}}\right)$ of the spectral-domain Green's function located on the proper Riemann sheet $\left(R_{n, i}\left(z, z^{\prime}\right)\right.$ are the residues of $\tilde{G}_{n}\left(k_{\rho}, z, z^{\prime}\right)$ at these poles), and the continuous spectrum part

$G_{n}^{\mathrm{CS}}\left(\rho, z, z^{\prime}\right)=\frac{1}{4 \pi} \int_{C_{b}} \tilde{G}_{n}\left(k_{\rho}, z, z^{\prime}\right) H_{n}^{(2)}\left(k_{\rho} \rho\right) k_{\rho}{ }^{n+1} \mathrm{~d} k_{\rho}$

corresponds to the integral along the Sommerfeld branch cut.

From a physical point of view, the above treatment can be viewed as a decomposition of the Green's function into a series of surface waveguide modes plus an additional term (here called the spatial wave) accounting for the reactive and/or radiating fields. The surface wave contribution gives rise to an asymptotic behavior for large $\rho$ of the type

$$
f_{\mathrm{sfw}}^{\infty}(\rho) \sim \frac{\mathrm{e}^{-\mathrm{j} k_{i}^{\mathrm{sw}} \rho}}{\sqrt{\rho}} .
$$

The spatial wave has been associated with the branch cuts of the spectral-domain Green's function. For the case of the grounded layered medium considered in Fig. 1 (whose upper boundary is free space), the branch points are located at $\pm k_{0}$ (the free-space wavenumber) and, therefore, the spatial wave would 
radiate into free space. Its associated asymptotic behavior for large $\rho$ is given by [19], [26], [30], [31]

$$
f_{\mathrm{spw}}^{\infty}(\rho) \sim \frac{\mathrm{e}^{-\mathrm{j} k_{0} \rho}}{\rho^{2}} .
$$

If the layered medium of Fig. 1 is lossless, both asymptotic behaviors usually appear simultaneously and the dominant far-field behavior of the Green's functions is given by (8) (namely, by the set of surface waveguide modes above cutoff), thus masking the far-field behavior of the spatial wave. It is in these common situations where the procedure reported in [7] works properly. However, there are at least three situations as follows in which the surface wave terms of (8) do not determine the far-field behavior.

Case 1) Lossless materials when the spectral-domain Green's function does not have proper poles.

As pointed out in [5, Sec. 7.2.2], this case occurs to the $\tilde{K}_{x x}^{A}\left(k_{\rho}, z, z^{\prime}\right)$ function of the layered medium of Fig. 1 at low frequencies. This component of the vector-potential spectral Green's dyad only has poles associated with TE surface waves [1], and since all these surface waves have nonzero cutoff frequency, below the cutoff frequency of the dominant TE surface wave, $\tilde{K}_{x x}^{A}\left(k_{\rho}, z, z^{\prime}\right)$ does not have proper poles. Thus, the only singularities of this Green's function are the branch points at $\pm k_{0}$, which causes that its corresponding far-field behavior is determined by the spatial wave given in (9).

Case 2) Lossy materials [20].

In this case, the far-field behavior of the surface waves fades out because of the exponential attenuation factor that appears in the numerator of (8) (due to losses, now $k_{i}^{\mathrm{sw}}=\beta_{i}^{\mathrm{sw}}-\mathrm{j} \alpha_{i}^{\mathrm{sw}}$ ). As a consequence, the dominant far-field behavior is again determined by the spatial wave in (9), whose exponential term does not show attenuation (assuming the upper half-space is lossless).

Case 3) When a pole is very close to the branch point.

This situation occurs at those frequency ranges around the cutoff frequencies of the surface wave modes (and, in particular, around the cutoff frequency of the first TE mode in case 1). It should be reminded that the pole associated with a surface waveguide mode below cutoff is located in the improper Riemann sheet, and this pole makes an excursion on this sheet as frequency increases running to the branch point, through which it moves to the proper sheet [19], [32]-[35]. The presence of this pole in the neighborhood of the branch point gives rise to a sort of mixed far-field behavior, which is a combination of those in (8) and (9) [20].

The above three cases can more easily be grouped into two different cases, i.e.: i) the far-field behavior is dominated by the spatial wave (which includes previous Cases 1) and 2)] and ii) the far-field behavior is determined by the mixed contribution of surface waves and spatial wave mentioned in the previous Case 3 ). Although the above two cases are clearly

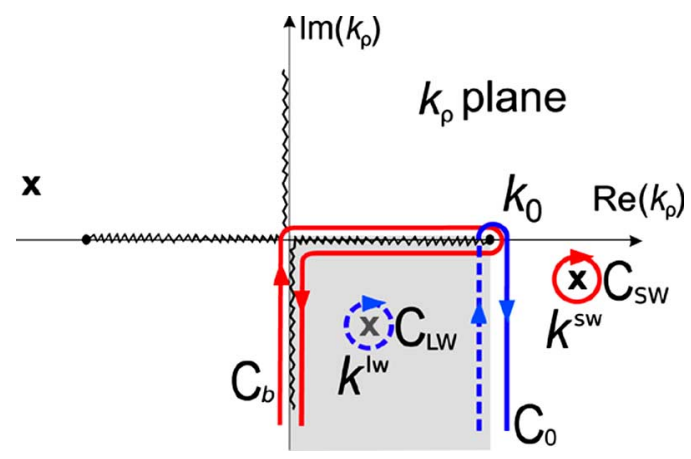

Fig. 3. Deformation of the integration path $C_{b}$. The improper pole $k_{1 \mathrm{w}}$ is captured in the deformation. The shadowed region is located on the improper sheet.

different, the former is a particular case of the latter since the spatial-wave far-field behavior can be viewed as a simplification of the mixed surface wave/spatial-wave far-field behavior. For this reason, in the following, only this latter situation will be considered in detail.

\section{B. Far-Field Behavior of the Residual Wave for Zeroth-Order Hankel Transforms}

As mentioned above, some of the possible problems to be found in the implementation of the rational function fitting procedure reported in [7] when dealing with "anomalous" cases are related to the approximation of the far-field behavior of the continuous-spectrum contribution of the Green's function. Thus, the following sections will present a detailed analysis of this subject, and how this problem can be handled to be incorporated in the rational function fitting procedure of [7]. For simplicity, this section will only consider the zeroth-order Hankel transform $(n=0)$, i.e.,

$$
G_{0}\left(\rho, z, z^{\prime}\right)=\frac{1}{4 \pi} \int_{-\infty(\mathrm{SIP})}^{\infty} \tilde{G}_{0}\left(k_{\rho}, z, z^{\prime}\right) H_{0}^{(2)}\left(k_{\rho} \rho\right) k_{\rho} \mathrm{d} k_{\rho}
$$

where function $\tilde{G}_{0}$ stands for any of the spectral-domain Green's functions $\tilde{K}_{\phi}, \tilde{K}_{x x}^{A}$, and $\tilde{K}_{z z}^{A}$ mentioned in [36, Form. C].

In order to simplify the problem, a further decomposition of the continuous-spectrum part of the Green's functions is suggested. This decomposition is based on a deformation of the integration path $C_{b}$ in (7) to the paths shown in Fig. 3 [35], [37] (the new path $C_{0}$ can be recognized as the steepest descent path when $z \equiv z^{\prime}=0$ [33]), which allows us to write

$$
G_{0}^{\mathrm{CS}}\left(\rho, z, z^{\prime}\right)=G_{0}^{\mathrm{RW}}\left(\rho, z, z^{\prime}\right)+G_{0}^{\mathrm{LW}}\left(\rho, z, z^{\prime}\right)
$$

where

$$
G_{0}^{\mathrm{RW}}\left(\rho, z, z^{\prime}\right)=\frac{1}{4 \pi} \int_{C_{0}} \tilde{G}_{0}\left(k_{\rho}, z, z^{\prime}\right) H_{0}^{(2)}\left(k_{\rho} \rho\right) k_{\rho} \mathrm{d} k_{\rho}
$$

will be denoted as the "residual wave" following the notation used in [37] (residual in the sense that it is the part of the Green's functions that is not accounted for by proper and improper 
modes), and

$$
\begin{aligned}
& G_{0}^{\mathrm{LW}}\left(\rho, z, z^{\prime}\right) \\
& \quad=\frac{1}{4 \pi} \sum_{m=1}^{N_{\mathrm{lw}}} \int_{C_{\mathrm{LW}, m}} \tilde{G}_{0}\left(k_{\rho}, z, z^{\prime}\right) H_{0}^{(2)}\left(k_{\rho} \rho\right) k_{\rho} \mathrm{d} k_{\rho} \\
& =-\frac{\mathrm{j}}{2} \sum_{m=1}^{N_{\mathrm{lw}}} R_{m}^{\mathrm{lw}}\left(z, z^{\prime}\right) H_{0}^{(2)}\left(k_{m}^{\mathrm{lw}} \rho\right) k_{m}^{\mathrm{lw}}
\end{aligned}
$$

with $R_{m}^{\mathrm{lw}}\left(z, z^{\prime}\right)$ being the residue of $\tilde{G}_{0}\left(k_{\rho}, z, z^{\prime}\right)$ at the $m$ th improper pole, $k_{m}^{\mathrm{lw}}\left(m=1, \ldots, N_{\mathrm{lw}}\right)$, located in the shadowed region of Fig. 2 (these improper poles are the only associated with physically meaningful leaky modes when $z \equiv z^{\prime}=0$ [19], [37]).

For large values of $\rho$, the asymptotic residual-wave contribution can be evaluated as

$$
\begin{aligned}
& G_{0}^{\mathrm{RW}, \infty}\left(\rho, z, z^{\prime}\right) \\
& \quad=\left.G_{0}^{\mathrm{RW}}\left(\rho, z, z^{\prime}\right)\right|_{\rho \gg} \\
& \left.\quad \approx \frac{1}{4 \pi} \int_{C_{0}} \tilde{G}_{0}\left(k_{\rho}, z, z^{\prime}\right) \sqrt{\frac{2 \mathrm{j}}{\pi k_{\rho} \rho}} \mathrm{e}^{-\mathrm{j} k_{\rho} \rho} k_{\rho} \mathrm{d} k_{\rho}\right|_{\rho \gg}
\end{aligned}
$$

where $H_{0}^{(2)}(\cdot)$ has been substituted by its asymptotic behavior [38]. Now following the procedure described in [37] and [39], after applying the change of variable $k_{\rho}=k_{0}-\mathrm{j} s$ to (14), the following expression is obtained:

$$
\begin{aligned}
& G_{0}^{\mathrm{RW}, \infty}\left(\rho, z, z^{\prime}\right)=\frac{-\mathrm{j}}{2 \pi} \sqrt{\frac{\mathrm{j}}{2 \pi}} \frac{\mathrm{e}^{-\mathrm{j} k_{0} \rho}}{\sqrt{\rho}} \\
& \times \int_{0}^{\infty}\left[\tilde{G}_{0}^{+}\left(s, z, z^{\prime}\right)-\tilde{G}_{0}^{-}\left(s, z, z^{\prime}\right)\right] \\
& \times\left.\sqrt{k_{0}-\mathrm{j} s} \mathrm{e}^{-s \rho} \mathrm{d} s\right|_{\rho \gg}
\end{aligned}
$$

where $\tilde{G}_{0}^{+}\left(s, z, z^{\prime}\right)\left(\tilde{G}_{0}^{-}\left(s, z, z^{\prime}\right)\right)$ represents the function $\tilde{G}_{0}\left(k_{\rho}=k_{0}-\mathrm{j} s, z, z^{\prime}\right)$ in the proper (improper) Riemann sheet. In order to obtain an asymptotic approximation of the integral of (15), the term multiplying $\mathrm{e}^{-s \rho}$ in the integrand is substituted by its limit as $s \rightarrow 0$ [37], [39], i.e.,

$$
\begin{aligned}
\int_{0}^{\infty} & {\left[\tilde{G}_{0}^{+}\left(s, z, z^{\prime}\right)-\tilde{G}_{0}^{-}\left(s, z, z^{\prime}\right)\right] } \\
& \times \sqrt{k_{0}-\left.\mathrm{j} s \mathrm{e}^{-s \rho} \mathrm{d} s\right|_{\rho \gg}} \\
\sim & \left.\sqrt{k_{0}} \int_{0}^{\infty}\left[\tilde{G}_{0}^{\left(k_{0}\right)}\left(s, z, z^{\prime}\right)\right]\right|_{s \rightarrow 0} \mathrm{e}^{-s \rho} \mathrm{d} s
\end{aligned}
$$

where

$$
\tilde{G}_{0}^{\left(k_{0}\right)}\left(s, z, z^{\prime}\right)=\tilde{G}_{0}^{+}\left(s, z, z^{\prime}\right)-\tilde{G}_{0}^{-}\left(s, z, z^{\prime}\right) .
$$

$\tilde{G}_{0}^{ \pm}\left(s, z, z^{\prime}\right)$ is now approximated in the neighborhood of $s \rightarrow 0$ by the following convenient quotient of first-order polynomials in $\sqrt{s}$ :

$$
\left.\tilde{G}_{0}^{ \pm}\left(s, z, z^{\prime}\right)\right|_{s \rightarrow 0} \approx \frac{C\left(z, z^{\prime}\right) \mp D\left(z, z^{\prime}\right) \sqrt{s}}{E\left(z, z^{\prime}\right) \mp F\left(z, z^{\prime}\right) \sqrt{s}}
$$

which makes

$$
\left.\tilde{G}_{0}^{\left(k_{0}\right)}\left(s, z, z^{\prime}\right)\right|_{s \rightarrow 0} \sim \frac{M_{p}\left(z, z^{\prime}\right) \sqrt{s}}{s-s_{p}\left(z, z^{\prime}\right)}
$$

where $M_{p}=2(D E-C F) / F^{2}$ and $s_{p}=(E / F)^{2}$. In the following, an implicit dependence on $z$ and $z^{\prime}$ will be always assumed in $M_{p}$ and $s_{p}$ (and then it will not be explicitly written). It should be noted that the denominator of (19) can properly account for the presence of a pole close to the branch point singularity (located at $s=0$ ). If there is not such a pole close to $k_{0}$, the behavior of $\tilde{G}_{0}^{\left(k_{0}\right)}\left(s, z, z^{\prime}\right)$ for small values of $s$ will be of the type $A_{p} \sqrt{s}$ [where $A_{p}=-M_{p} / s_{p}$ since $s$ will be negligible when compared with $s_{p}$ in the denominator of (19)].

From a numerical point of view, the coefficients $M_{p}$ and $s_{p}$ can be obtained through the following overdetermined system of linear equations:

$$
\left(s_{n}-s_{p}\right) \tilde{G}_{0}^{\left(k_{0}\right)}\left(s_{n}\right)=M_{p} \sqrt{s_{n}} \quad n=1,2, \ldots, N_{s}
$$

where the values of $s_{n}$ can be taken in logarithmic scale ranging, for example, from $s / k_{0}=10^{-8}$ to $s / k_{0}=10^{-1}$. The overdetermined system can be solved, for instance, by means of a total least squares approach (in our experience, $N_{s} \leq 8$ provided sufficient accuracy) [22]. For the particular case of a grounded dielectric slab when the field and point sources are located at the air-dielectric interface (namely, $z \equiv z^{\prime}=0$ in Fig. 1), Appendix I provides closed-form expressions for $M_{p}$ and $s_{p}$ for the different relevant Green's functions.

When (16) and (19) are introduced in (15), and $s_{p}$ is not in the fourth quadrant of the complex plane (as should be found for any physical situation), it is found that (see [39, App.])

$$
\begin{aligned}
G_{0}^{\mathrm{RW}, \infty} & \left(\rho, z, z^{\prime}\right) \\
\sim & \frac{-\mathrm{j} M_{p}}{2 \pi} \sqrt{\frac{j k_{0}}{2 \pi}} \frac{\mathrm{e}^{-\mathrm{j} k_{0} \rho}}{\sqrt{\rho}} \int_{0}^{\infty} \frac{\sqrt{s} \mathrm{e}^{-s \rho}}{s-s_{p}} \mathrm{~d} s \\
= & \frac{-\mathrm{j} M_{p}}{2 \pi} \sqrt{\frac{\mathrm{j} k_{0}}{2 \pi}} \frac{\mathrm{e}^{-\mathrm{j} k_{0} \rho}}{\sqrt{\rho}} \\
& \times\left\{\sqrt{\frac{\pi}{\rho}}+\mathrm{j} \pi \sqrt{s_{p}} \mathrm{e}^{-s_{p} \rho} \operatorname{erfc}\left(-\mathrm{j} \sqrt{s_{p} \rho}\right)\right\}
\end{aligned}
$$

where $\operatorname{erfc}(\cdot)$ is the complementary error function. In order to account for the effect of the residual wave in the spectral Green's functions expansions of (1) and (2), it would be very convenient to incorporate the spectral-domain version of $G_{0}^{\mathrm{RW}, \infty}\left(\rho, z, z^{\prime}\right)$ in these equations in an explicit way before applying the method of total least squares. However, to the best of our knowledge, the Hankel transform of the function $G_{0}^{\mathrm{RW}, \infty}\left(\rho, z, z^{\prime}\right)$, shown in (21), cannot be obtained in closed form. Using the asymptotic expansion of the complementary error function of complex argument $\operatorname{erfc}(z)$ for $|z| \rightarrow \infty$ [38, eq. (7.123)], it is possible to show that the function $G_{0}^{\mathrm{RW}, \infty}\left(\rho, z, z^{\prime}\right)$ of $(21)$ can be written for large $\rho$ in two different ways depending on the value of $\left|s_{p} \rho\right|$ (see the comments following [31, eq. (21)]), namely,

$$
\begin{aligned}
& G_{0}^{\mathrm{RW}, \infty}\left(\rho, z, z^{\prime}\right) \\
& \quad \sim \frac{-\mathrm{j} M_{p}}{2 \pi} \sqrt{\frac{\mathrm{j} k_{0}}{2} \frac{\mathrm{e}^{-\mathrm{j} k_{0} \rho}}{\rho}} \times \begin{cases}1, & \left|s_{p} \rho\right| \ll 1 \\
\frac{-1}{2 s_{p} \rho}, & \left|s_{p} \rho\right| \gg 1 .\end{cases}
\end{aligned}
$$

The upper term of (22) matches the asymptotic behavior of $G_{0}^{\mathrm{RW}, \infty}\left(\rho, z, z^{\prime}\right)$ in a wide range of values of $\rho$ when the pole $k_{p}=k_{0}-\mathrm{j} s_{p}$ of $\tilde{G}_{0}\left(k_{\rho}, z, z^{\prime}\right)$ is very close to the branch point at $k_{0}$ (i.e., when $\left|s_{p} / k_{0}\right| \ll 1$ ), and the lower term of (22) 
dominates the asymptotic behavior when the pole is far from the branch point $\left(\left|s_{p} / k_{0}\right| \gtrsim 1\right)$. In order to combine the two asymptotic behaviors of (22) in a single expression, we propose the use of the following far-field (ff) asymptotic behavior of $G_{0}^{\mathrm{RW}, \infty}\left(\rho, z, z^{\prime}\right)$ :

$$
\begin{aligned}
G_{0}^{\mathrm{RW}, \mathrm{ff}}\left(\rho, z, z^{\prime}\right)=- & \frac{\mathrm{j} M_{p}}{2 \pi} \sqrt{\frac{\mathrm{j} k_{0}}{2}} \frac{\mathrm{e}^{-\mathrm{j} k_{0} \rho}\left(1-\mathrm{e}^{-k_{0} \rho}\right)^{2}}{\rho} \\
& \times\left\{\sum_{i=1}^{N_{\mathrm{py}}} A_{i}^{\mathrm{py}} \mathrm{e}^{-b_{i}^{\mathrm{py}}\left|s_{p}\right| \rho}-\frac{1-\mathrm{e}^{-\left|s_{p}\right| \rho}}{2 s_{p} \rho}\right\}
\end{aligned}
$$

which fulfills the conditions of (22) for $\left|s_{p} \rho\right| \ll 1$ and $\left|s_{p} \rho\right| \gg 1$ (assuming $\rho \gg$ ) provided $\sum_{i=1}^{N_{\text {py }}} A_{i}^{\text {py }}-\left(\left|s_{p}\right| / 2 s_{p}\right)=1$ and $\operatorname{Re}\left(b_{i}^{\mathrm{py}}\right)>0\left(i=1, \ldots, N_{\mathrm{py}}\right)$. The unknown coefficients $A_{i}^{\mathrm{py}}$ and $b_{i}^{\text {py }}$ have to be chosen in such a way that $G_{0}^{\mathrm{RW}, \mathrm{ff}}\left(\rho, z, z^{\prime}\right)$ is a good fitting of $G_{0}^{\mathrm{RW}, \infty}\left(\rho, z, z^{\prime}\right)$ for large $\rho$. According to (21) and (23), this can be accomplished when the following condition is satisfied:

$$
\begin{aligned}
1+\mathrm{j} \sqrt{\pi s_{p} \rho} \mathrm{e}^{-s_{p} \rho} \operatorname{erfc}\left(-\mathrm{j} \sqrt{s_{p} \rho}\right)+ & \frac{1-\mathrm{e}^{-\left|s_{p}\right| \rho}}{2 s_{p} \rho} \\
& \approx \sum_{i=1}^{N_{\mathrm{py}}} A_{i}^{\mathrm{py}} \mathrm{e}^{-b_{i}^{\mathrm{py}}\left|s_{p}\right| \rho} .
\end{aligned}
$$

In this paper, we have obtained $A_{i}^{\mathrm{py}}$ and $b_{i}^{\mathrm{py}}\left(i=1, \ldots, N_{\mathrm{py}}\right)$ by enforcing that (24) is exactly satisfied at the samples $\rho_{i}=(i-1) \rho_{\max } /\left(2 N_{\mathrm{py}}-1\right)\left(i=1, \ldots, 2 N_{\mathrm{py}}\right)$, and by applying Prony's method [24]. Numerical simulations have shown that when we choose $\rho_{\max }=80 / \sqrt{\left|k_{p} s_{p}\right|}$ and $N_{\text {py }}=5$, the values obtained for $A_{i}^{\mathrm{py}}$ and $b_{i}^{\mathrm{py}}\left(i=1, \ldots, N_{\mathrm{py}}\right)$ ensure that $G_{0}^{\mathrm{RW}, \mathrm{ff}}\left(\rho, z, z^{\prime}\right)$ is a good fitting of $G_{0}^{\mathrm{RW}, \infty}\left(\rho, z, z^{\prime}\right)$ in the far field.

The term $\left(1-\mathrm{e}^{-k_{0} \rho}\right)^{2}$ has been introduced in (23) to avoid that $G_{0}^{\mathrm{RW}, \mathrm{ff}}\left(\rho, z, z^{\prime}\right)$ contributes the near field of $G_{0}\left(\rho, z, z^{\prime}\right)$ (in fact, whereas $G_{0}\left(\rho, z, z^{\prime}\right) \propto \rho^{-1}$ as $\rho \rightarrow 0$ when $z=z^{\prime}$, and $G_{0}\left(\rho, z, z^{\prime}\right) \propto \rho^{0}$ as $\rho \rightarrow 0$ when $z \neq z^{\prime}$ [7], it turns out that $G_{0}^{\mathrm{RW}, \mathrm{ff}}\left(\rho, z, z^{\prime}\right) \propto \rho$ as $\left.\rho \rightarrow 0\right)$. It should be noted that the key advantage of (23) over (21) is that the spectral-domain counterpart of (23) can be obtained in closed form, and is given by

$$
\begin{aligned}
& \tilde{G}_{0}^{\mathrm{RW}, \mathrm{ff}}\left(k_{\rho}, z, z^{\prime}\right) \\
& =2 \pi \int_{0}^{\infty} G_{0}^{\mathrm{RW}, \mathrm{ff}}\left(\rho, z, z^{\prime}\right) J_{0}\left(k_{\rho} \rho\right) \rho \mathrm{d} \rho \\
& =-\mathrm{j} M_{p} \sqrt{\frac{\mathrm{j} k_{0}}{2}}\left\{\sum _ { i = 1 } ^ { N _ { \mathrm { py } } } A _ { i } ^ { \mathrm { py } } \left[S\left(k_{\rho}, b_{i}^{\mathrm{py}}\left|s_{p}\right|+\mathrm{j} k_{0}\right)\right.\right. \\
& -2 S\left(k_{\rho}, b_{i}^{\mathrm{py}}\left|s_{p}\right|+(\mathrm{j}+1) k_{0}\right) \\
& \left.+S\left(k_{\rho}, b_{i}^{\mathrm{py}}\left|s_{p}\right|+(\mathrm{j}+2) k_{0}\right)\right] \\
& -\frac{1}{2 s_{p}}\left[L\left(k_{\rho}, \mathrm{j} k_{0}\right)-2 L\left(k_{\rho},(\mathrm{j}+1) k_{0}\right)\right. \\
& \left.\left.+L\left(k_{\rho},(\mathrm{j}+2) k_{0}\right)\right]\right\}
\end{aligned}
$$

with

$$
\begin{aligned}
& S\left(k_{\rho}, x\right)=\left[\sqrt{k_{\rho}{ }^{2}+x^{2}}\right]^{-1} \\
& L\left(k_{\rho}, x\right)=\ln \left[\frac{x+\left|s_{p}\right|+\sqrt{k_{\rho}^{2}+\left(\left|s_{p}\right|+x\right)^{2}}}{x+\sqrt{k_{\rho}^{2}+x^{2}}}\right] .
\end{aligned}
$$

In case the pole $s_{p}$ of (19) gives place to a pole in the $k_{\rho}$ plane, $k_{p}=k_{0}-\mathrm{j} s_{p}$, that explicitly contributes to the final expression of $G_{0}\left(\rho, z, z^{\prime}\right)$ (either because it is a proper pole contained in the discrete spectrum of (6) or because it is an improper pole located in the shadowed region of Fig. 3), numerical simulations have shown that this pole should be explicitly incorporated in the Green's functions expansions of (1) and (2) before applying the method of total least squares. This can easily be done by adding the following contribution in the spatial domain:

$$
G_{0}^{(\mathrm{p})}\left(\rho, z, z^{\prime}\right)=-\frac{\mathrm{j}}{2} R_{p} k_{p} H_{0}^{(2)}\left(k_{p} \rho\right)
$$

while its spectral-domain counterpart is given by

$$
\tilde{G}_{0}^{(\mathrm{p})}\left(k_{\rho}, z, z^{\prime}\right)=\frac{2 R_{p} k_{p}}{k_{\rho}^{2}-k_{p}^{2}}
$$

where

$$
\begin{aligned}
R_{p} & =\lim _{k_{\rho} \rightarrow k_{p}}\left(k_{\rho}-k_{p}\right) \tilde{G}_{0}\left(k_{\rho}, z, z^{\prime}\right) \\
& =\mp \mathrm{j} \lim _{s \rightarrow s_{p}}\left(s-s_{p}\right) \tilde{G}_{0}^{\left(k_{0}\right)}\left(s, z, z^{\prime}\right)=\mp \mathrm{j} M_{p} \sqrt{s_{p}} .
\end{aligned}
$$

In (30), the minus sign must be chosen when $k_{p}$ is proper and the plus sign must be chosen when $k_{p}$ is improper. The proper/improper nature of $k_{p}$ can readily be deduced from the numerical analysis of the sign of

$$
B_{p}=\left|\tilde{G}_{0}^{+}\left(s_{p}(1+\epsilon), z, z^{\prime}\right)\right|-\left|\tilde{G}_{0}^{-}\left(s_{p}(1+\epsilon), z, z^{\prime}\right)\right|
$$

with $\epsilon=10^{-10}$. In fact, $k_{p}$ turns out to be proper if $B_{p}>0$ and improper if $B_{p}<0$.

As commented in [7], the Hankel function $H_{0}^{(2)}\left(k_{p} \rho\right)$ of (28) presents a logarithmic singularity when $\rho=0$ that may deteriorate the near-field approximation of $G_{0}^{(\mathrm{p})}\left(\rho, z, z^{\prime}\right)$, especially when $z \neq z^{\prime}$ (see [7, Fig. 8]). In order to eliminate this near-field problem, we have followed the approach of [31, eq. (8)] and substituted the pole contributions of (28) and (29) by the following corrected pole (cp) contributions:

$$
\begin{aligned}
G_{0}^{(\mathrm{cp})}\left(\rho, z, z^{\prime}\right) & =-\frac{\mathrm{j}}{2} R_{p} k_{p}\left[H_{0}^{(2)}\left(k_{p} \rho\right)-H_{0}^{(2)}(-\mathrm{j} c \rho)\right] \\
\tilde{G}_{0}^{(\mathrm{cp})}\left(k_{\rho}, z, z^{\prime}\right) & =\frac{2 R_{p} k_{p}\left(c^{2}+k_{p}^{2}\right)}{\left(k_{\rho}{ }^{2}-k_{p}^{2}\right)\left(k_{\rho}{ }^{2}+c^{2}\right)}
\end{aligned}
$$

where $c$ must be a large positive real number. In particular, we have chosen $c=10 k_{0}$. 
The Hankel function $H_{0}^{(2)}(-\mathrm{j} c \rho)$ introduced in (31) is an annihilating function that both prevents $G_{0}^{(\mathrm{cp})}\left(\rho, z, z^{\prime}\right)$ from being singular as $\rho \rightarrow 0$ and makes it possible that $G_{0}^{(\mathrm{cp})}\left(\rho, z, z^{\prime}\right) \approx$ $G_{0}^{(\mathrm{p})}\left(\rho, z, z^{\prime}\right)$ when $\rho \gg[31]$. Note that $\tilde{G}_{0}^{(\mathrm{cp})}\left(k_{\rho}, z, z^{\prime}\right)$ decays like $k_{\rho}{ }^{-4}$ for large $k_{\rho}$, which is to be expected for the zeroth-order Hankel transform of a surface wave term, which does not lead to nonphysical near-field singularities in the spatial domain [7].

\section{Far-Field Behavior of the Residual Wave for First-Order Hankel Transforms}

For the spectral Green's function $\tilde{G}_{1}$ (here, $\tilde{G}_{1}$ stands for the functions $\tilde{K}_{z x}^{A} / \mathrm{j} k_{x}, \tilde{K}_{z y}^{A} / \mathrm{j} k_{y}, \tilde{K}_{x z}^{A} / \mathrm{j} k_{x}$, and $\tilde{K}_{y z}^{A} / \mathrm{j} k_{y}$ in [36, Form. C]), the inverse Hankel transform to be evaluated is given by

$$
G_{1}\left(\rho, z, z^{\prime}\right)=\frac{1}{4 \pi} \int_{-\infty(\operatorname{SIP})}^{\infty} \tilde{G}_{1}\left(k_{\rho}, z, z^{\prime}\right) H_{1}^{(2)}\left(k_{\rho} \rho\right) k_{\rho}^{2} \mathrm{~d} k_{\rho}
$$

where the integration path is the same as that used in (10).

Following the same rationale as in Section II-B, it is possible to show that the asymptotic behavior of the corresponding residual wave is given by

$$
\begin{aligned}
G_{1}^{\mathrm{RW}, \infty}\left(\rho, z, z^{\prime}\right) \sim \frac{M_{p} k_{0}}{2 \pi} \sqrt{\frac{\mathrm{j} k_{0}}{2 \pi}} \frac{\mathrm{e}^{-\mathrm{j} k_{0} \rho}}{\sqrt{\rho}} \\
\times\left\{\sqrt{\frac{\pi}{\rho}}+\mathrm{j} \pi \sqrt{s_{p}} \mathrm{e}^{-s_{p} \rho} \operatorname{erfc}\left(-\mathrm{j} \sqrt{s_{p} \rho}\right)\right\}
\end{aligned}
$$

where $M_{p}$ and $s_{p}$ are computed by means of (20), but in this case, $\tilde{G}_{0}^{\left(k_{0}\right)}\left(s_{n}\right)$ has to be substituted by $\tilde{G}_{1}^{\left(k_{0}\right)}\left(s_{n}\right)$.

Since the Hankel transform of $G_{1}^{\mathrm{RW}, \infty}\left(\rho, z, z^{\prime}\right)$ cannot be obtained in closed form, the following alternative far-field behavior with closed-form Hankel transform is proposed for the residual wave [see (23)]

$$
\begin{aligned}
G_{1}^{\mathrm{RW}, \mathrm{ff}}\left(\rho, z, z^{\prime}\right)=\frac{M_{p} k_{0}}{2 \pi} \sqrt{\frac{\mathrm{j} k_{0}}{2}} \frac{\mathrm{e}^{-\mathrm{j} k_{0} \rho}\left(1-\mathrm{e}^{-k_{0} \rho}\right)^{3}}{\rho} \\
\times\left\{\sum_{i=1}^{N_{\mathrm{py}}} A_{i}^{\mathrm{py}} \mathrm{e}^{-b_{i}^{\mathrm{py}}\left|s_{p}\right| \rho}-\frac{1-\mathrm{e}^{-\left|s_{p}\right| \rho}}{2 s_{p} \rho}\right\} .
\end{aligned}
$$

As happens in (23), the term $\left(1-\mathrm{e}^{-k_{0} \rho}\right)^{3}$ has been introduced in (35) to avoid that $G_{1}^{\mathrm{RW}, \mathrm{ff}}\left(\rho, z, z^{\prime}\right)$ masks the near-field behavior of $G_{1}\left(\rho, z, z^{\prime}\right)$ (in fact, whereas for most values of $z$ and $z^{\prime}, G_{1}\left(\rho, z, z^{\prime}\right) \propto \rho$ as $\rho \rightarrow 0$, it turns out that $G_{1}^{\mathrm{RW}, \mathrm{ff}}\left(\rho, z, z^{\prime}\right) \propto \rho^{2}$ as $\left.\rho \rightarrow 0\right)$. The spectral-domain counterpart of (35) is given in Appendix II.

If the pole $s_{p}$ explicitly contributes to $G_{1}\left(\rho, z, z^{\prime}\right)$, then it should be incorporated in (1) and (2) as discussed in Section II-B. In the present case, the corresponding corrected pole contribution to be added in the spatial domain is

$$
\begin{aligned}
G_{1}^{(\mathrm{cp})}\left(\rho, z, z^{\prime}\right) & \\
=-\frac{\mathrm{j}}{2} R_{p} k_{p} & {\left[k_{p} H_{1}^{(2)}\left(k_{p} \rho\right)+\mathrm{j} d \frac{e^{2}+k_{p}^{2}}{e^{2}-d^{2}} H_{1}^{(2)}(-\mathrm{j} d \rho)\right.} \\
& \left.-\mathrm{je} \frac{d^{2}+k_{p}^{2}}{e^{2}-d^{2}} H_{1}^{(2)}(-\mathrm{j} e \rho)\right]
\end{aligned}
$$

where $R_{p}$ is the residue of $\tilde{G}_{1}\left(k_{\rho}, z, z^{\prime}\right)$ at $k_{\rho}=k_{p}$, and $d$ and $e$ must be two different large positive real numbers, which have been chosen as $d=10 k_{0}$ and $e=12 k_{0}$. Owing to the two annihilating Hankel functions $H_{1}^{(2)}(-\mathrm{j} d \rho)$ and $H_{1}^{(2)}(-\mathrm{j} e \rho)$, it turns out that $G_{1}^{(\mathrm{cp})}\left(\rho, z, z^{\prime}\right) \propto \rho$ as $\rho \rightarrow 0$, and this avoids that the singularity of $H_{1}^{(2)}\left(k_{p} \rho\right)$ introduces a nonphysical behavior in the approximation of $G_{1}\left(\rho, z, z^{\prime}\right)$ (see [7, Fig. 10]). The spectral-domain counterpart of (36) is given by

$$
\tilde{G}_{1}^{(\mathrm{cp})}\left(k_{\rho}, z, z^{\prime}\right)=\frac{2 R_{p} k_{p}\left[d^{2} e^{2}+k_{p}^{2}\left(d^{2}+e^{2}+k_{p}^{2}\right)\right]}{\left(k_{\rho}{ }^{2}-k_{p}^{2}\right)\left(k_{\rho}{ }^{2}+d^{2}\right)\left(k_{\rho}{ }^{2}+e^{2}\right)} .
$$

Note that $\tilde{G}_{1}^{(\mathrm{cp})}\left(k_{\rho}, z, z^{\prime}\right)$ decays like $k_{\rho}{ }^{-6}$ for large $k_{\rho}$, which is the behavior expected for the first-order Hankel transform of a surface wave term, which does not lead to a nonphysical near-field behavior in the spatial domain [7].

\section{Application of the Total Least Square Procedure}

Once the far-field behavior of both the residual wave and the contribution of the pole $s_{p}$ have been estimated in closed form, both in the spatial and spectral domains, their spectral-domain versions can be added to the approximations of (2) in the following way:

$$
\begin{aligned}
\tilde{G}_{n}\left(k_{\rho}, z, z^{\prime}\right) \approx & \tilde{G}_{n}^{\mathrm{as}}\left(k_{\rho}, z, z^{\prime}\right) \\
& +\tilde{G}_{n}^{\mathrm{RW}, \mathrm{ff}}\left(k_{\rho}, z, z^{\prime}\right)+\tilde{G}_{n}^{(\mathrm{cp})}\left(k_{\rho}, z, z^{\prime}\right) \\
& +\tilde{G}_{n}^{\mathrm{TLS}(\mathrm{pr})}\left(k_{\rho}, z, z^{\prime}\right), \quad n=0,1 .
\end{aligned}
$$

This means that the total least squares procedure reported in [7] can now properly deal with "anomalous" cases, thus making it possible to cover most of the physical situations encountered in practice. The corresponding spatial-domain counterpart of (38) can be given in the following quasi-analytical way:

$$
\begin{aligned}
& G_{n}\left(\rho, z, z^{\prime}\right) \\
& \approx G_{n}^{\mathrm{as}}\left(\rho, z, z^{\prime}\right) \\
& \quad+G_{n}^{\mathrm{RW}, \mathrm{ff}}\left(\rho, z, z^{\prime}\right)+G_{n}^{(\mathrm{cp})}\left(\rho, z, z^{\prime}\right) \\
& \quad-\frac{\mathrm{j}}{4} \sum_{i=1}^{M} a_{n, i}\left(p_{n, i}\right)^{n} H_{n}^{(2)}\left(p_{n, i} \rho\right), \quad n=0,1
\end{aligned}
$$

where it has been possible to express $G_{n}\left(\rho, z, z^{\prime}\right)$ in closed form in terms of known functions. These functions depend on a number of coefficients that can easily be determined via the methods of Prony and total least squares with very low computational effort. It should be pointed out that, apart from the term $\tilde{G}_{n}^{(\mathrm{cp})}\left(k_{\rho}, z, z^{\prime}\right)$ of (38) related to the pole $k_{p}=k_{0}-\mathrm{j} s_{p}$, the surface wave modes associated with the proper poles that appear in 
the discrete spectrum of the Green's functions [see (6)], as well as the leaky wave modes related to improper poles that appear in the continuous spectrum of the Green's functions [see (13)] would be well accounted for by the pole-residue expansion of (38) via the method of total least squares [7], and no extra effort should be carried out to incorporate these terms explicitly in (38).

From a computational point of view, the inclusion of the continuous spectrum only represents a computational overload of $20 \%$ at most when compared with the "simplified" total least squares approach reported in [7].

\section{NUMERICAL RESULTS}

In this section, the theory presented above will be validated in situations where the far field of the Green's functions is dominated by surface waves (the far field is given by (8)) and in "anomalous" situations where the far field is dominated by the continuous spectrum [the far field is given by (9)]. For this purpose, on the one hand, the exact (e) values of the spectral-domain function

$$
\begin{aligned}
\tilde{G}_{n}^{\mathrm{TLS}(\mathrm{e})}\left(k_{\rho}, z, z^{\prime}\right)=\tilde{G}_{n}\left(k_{\rho}, z, z^{\prime}\right)-\tilde{G}_{n}^{\mathrm{as}}\left(k_{\rho}, z, z^{\prime}\right) \\
\quad-\tilde{G}_{n}^{\mathrm{RW}, \mathrm{ff}}\left(k_{\rho}, z, z^{\prime}\right)-\tilde{G}_{n}^{(\mathrm{cp})}\left(k_{\rho}, z, z^{\prime}\right)
\end{aligned}
$$

will be compared with the values of the fitting spectral-domain function $\tilde{G}_{n}^{\mathrm{TLS}(\mathrm{pr})}\left(k_{\rho}, z, z^{\prime}\right)$ of (1) obtained via the method of total least squares. On the other hand, the approximate values of the spatial-domain Green's functions provided by (39) will be compared with numerical data obtained by a direct numerical integration of Sommerfeld integrals.

As a first example, Fig. 4 shows results for the scalar potential Green's function of a grounded dielectric slab in the particular case reported in ([7, Figs. 3 and 4]). This figure shows that the new approach described in Section II works as efficiently as that presented by the authors in [7] both in the spectral and spatial domains. The case studied in Fig. 4 was also treated in [20, Fig. 6(a)], where it was pointed out that the presence of a proper pole very close to the branch point $k_{0}$ (this pole, $k_{2}^{\mathrm{sw}} / k_{0}=1.0000271$, is associated with the first TE mode of the grounded dielectric slab) influences the far field of the continuous spectrum. However, this case was successfully dealt with by the theory presented in [7] because the scalar potential Green's function presents another proper pole that is not close to the branch point (this pole, $k_{1}^{\mathrm{sw}} / k_{0}=1.4792905$, is associated with the dominant TM mode of the grounded dielectric slab). Since the surface wave associated to this latter pole dominates the far field of $K_{\phi}$, which is of the type $\rho^{-1 / 2}$, the contribution of the continuous spectrum to the far field of $K_{\phi}$ is masked in this case and the approach of [7] works without a problem. Owing to the fact that the far field of $K_{\phi}$ is dominated by a surface wave, one expects that the contribution of the continuous spectrum does not have to be explicitly incorporated when approximating $K_{\phi}$ via the method of total least squares. Anyway, the results of Fig. 4(b) make apparent that the inclusion of the continuous spectrum, as in (39), does not affect the good performance of the method of total least squares (in fact, the relative

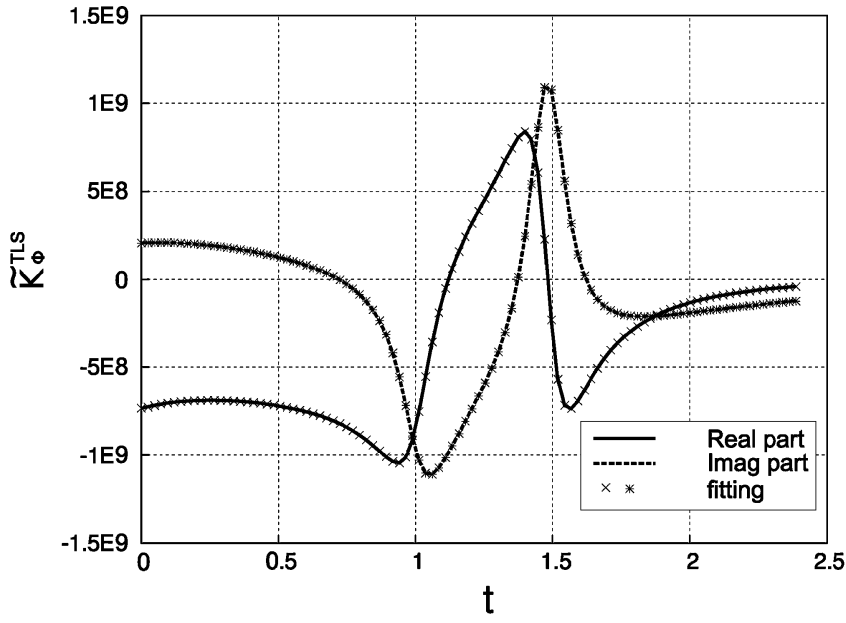

(a)

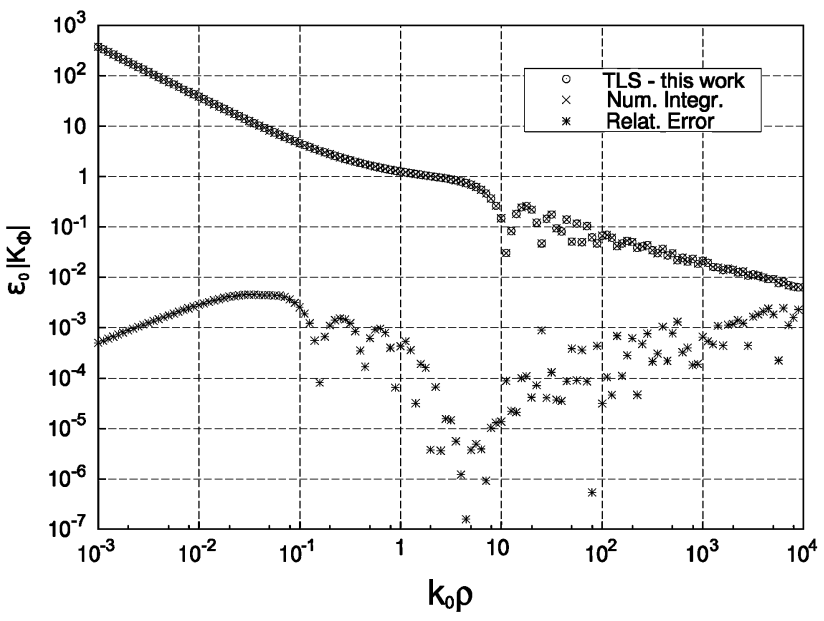

(b)

Fig. 4. (a) Real and imaginary parts of the spectral-domain functions $\tilde{K}_{\phi}^{\mathrm{TLS}(\mathrm{e})}$ (solid and dashed lines) and $\tilde{K}_{\phi}^{\text {TLS}(p r)}(\times, *)$ along path $C$ of [7, Fig. 1]. Maximum relative error: $\leq 0.005$. (b) Magnitude of the spatial-domain Green's function $K_{\phi}$. Numerical integration results $(+)$ are compared with those obtained via (39) $(\odot)$. The relative error $(x)$ between these two sets of results is also shown. Parameters of the total least squares (TLS): $A=0.1, T_{0}=$ 2.2, $M=12, N=27$ [7]. Parameters of the structure: $f=4.075 \mathrm{GHz}$, $z^{\prime}=z=0, N_{\mathrm{la}}=1 \varepsilon_{r 1}=4.4, \tan \delta_{1}=0, h_{1}=10 \mathrm{~mm}$.

error in the spatial domain is below $0.4 \%$ along all the seven decades shown).

In Fig. 5, we plot the $K_{x x}^{A}$ Green's function of the grounded dielectric slab of Fig. 4 at a lower frequency $(f=3 \mathrm{GHz}$ ) where no TE modes are above cutoff. The situation studied in Fig. 5 is very different from that studied in Fig. 4. Since $\tilde{K}_{x x}^{A}$ only has poles associated with TE modes, at $3 \mathrm{GHz}$ the only singularities of this spectral Green's function in the proper Riemann sheet are the pair of branch points at $\pm k_{0}$. Specifically in Fig. 5(a), the fitted values provided by the method of total least squares are compared with the exact values when both the approach of this paper and the approach of [7] are followed. Both fittings via the method of total least squares have given excellent results (the maximum relative error for both curves is less than $0.5 \%$ ). However, Fig. 5(b) shows that whereas the results obtained for $K_{x x}^{A}$ via the present approach (circles) match well with the results obtained by direct numerical integration within the considered 


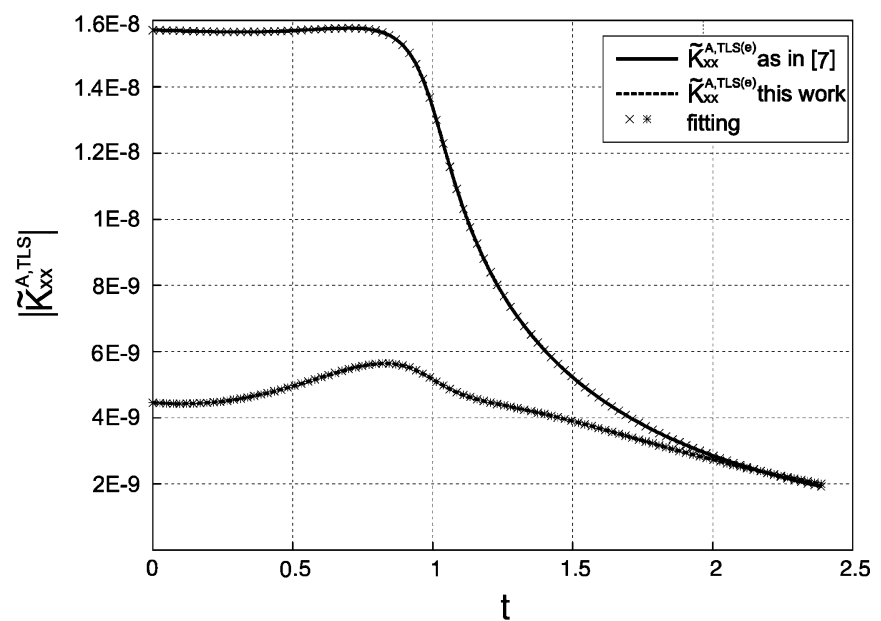

(a)

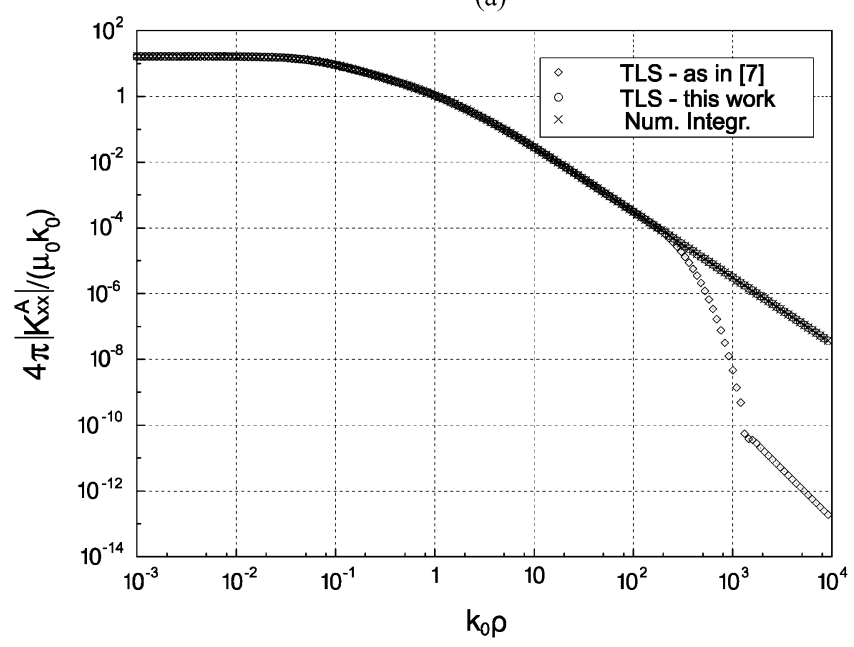

(b)

Fig. 5. (a) Magnitude of the spectral-domain functions $\tilde{K}_{x x}^{A, T L S(e)}$ and $\tilde{K}_{x x}^{A, T L S(p r)}$ along path $C$ of [7, Fig. 1]. The solid line and (*) represent the total least squares fitting followed in [7, eq. (6)]. The dashed line and $(x)$ represent the approach followed in this paper [see (1) and (40)]. Maximum relative error: $\leq$ 0.003. (b) Magnitude of the spatial-domain Green's function $K_{x x}^{A}$. Numerical integration results $(\times)$ are compared with those obtained via [7, eq. (13)] $(\diamond)$ and with those obtained via (39) $(\odot)$. Maximum relative error between $\times$ and $\odot$ is smaller than 0.005. Parameters of the total least squares (TLS): $A=0.1, T_{0}=2.4, M=12$, and $N=27$. Parameters of the structure: $f=3 \mathrm{GHz}, z^{\prime}=0.5 \mathrm{~mm}, z=-0.5 \mathrm{~mm}$, $N_{\mathrm{la}}=1 ; \varepsilon_{r 1}=4.4, \tan \delta_{1}=0, h_{1}=10 \mathrm{~mm}$.

range of values of $\rho$ (the maximum relative error between these two set of results is less than $0.5 \%$ ), the results provided by the procedure of [7] (diamonds) are only valid for the near and intermediate field zones despite the good fitting provided by the method of total least squares in the spectral domain. Certainly, in this case, the method of [7] cannot give reasonable results in the far field since the contribution of the continuous spectrum dominates the far field, and this contribution is not considered in the approach of [7]. It is interesting to note that whereas the results of Fig. 5(b) obtained with the current approach predict a correct far-field behavior of the type $\rho^{-2}$ for $K_{x x}^{A}$ [see (9)] thanks to the contribution of $K_{x x}^{A, \mathrm{RW}, \mathrm{ff}}\left(\rho, z, z^{\prime}\right)$, the approach of [7] predicts a far-field behavior of the type $\rho^{-3}$ for $K_{x x}^{A}$, which is supplied by the quasi-static term $K_{x x}^{A \text {,as }}\left(\rho, z, z^{\prime}\right)$ (see [7, eq. (45)]) and is clearly wrong.

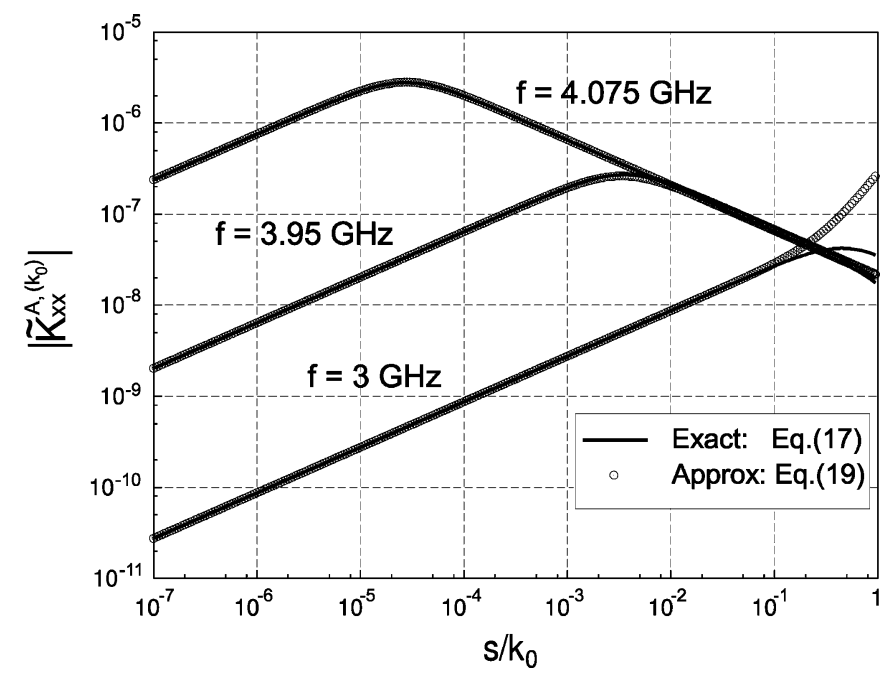

Fig. 6. Magnitude of the spectral-domain function $\tilde{K}_{x x}^{A\left(k_{0}\right)}$ defined in (17) as a function of the normalized real variable $s / k_{0}$. The exact values of $\tilde{K}_{x x}^{A\left(k_{0}\right)}$ (solid line) are compared with the values obtained via the approximation of (19) $(\odot)$, which is valid as $s \rightarrow 0$. Parameters as in Fig. 5 .

In Fig. 6, the spectral-domain function $\tilde{K}_{x x}^{A,\left(k_{0}\right)}\left(s, z, z^{\prime}\right)$ defined in (17) is plotted at three different frequencies for the structure analyzed in Fig. 5. It should be noted that $\tilde{K}_{x x}^{A,\left(k_{0}\right)}\left(s, z, z^{\prime}\right)$ is well fitted by the single pole approximation given in (19) provided that $s / k_{0} \lesssim 10^{-1}$. The structure of Fig. 5 is again analyzed in Fig. 7, where the values of $K_{x x}^{A}$ are plotted in a wide range of distances at the three frequencies analyzed in Fig. 6. A very good agreement is found in Fig. 7 between the results obtained through the approach of Section II and the results obtained by numerical integration for the three frequencies considered. The strong correlation that exists between the results of Fig. 6 and the results of Fig. 7 will be discussed in detail below. A similar discussion was reported in [40, Fig. 15] in connection with the excitation of the continuous spectrum in microstrip lines.

For the structure studied in Fig. 5 , at $f=3 \mathrm{GHz}$, the value obtained for $s_{p} / k_{0}$ after solving (20) is far from zero, and the pole $k_{p}=k_{0}-\mathrm{j} s_{p}$ is also far from the branch point $k_{0}$. It causes that the behavior of $\tilde{K}_{x x}^{A,\left(k_{0}\right)}\left(s, z, z^{\prime}\right)$ observed in Fig. 6 for small $s$ is of the type $\sqrt{s}$ [s will be negligible when compared with $s_{p}$ in the denominator of the approximation in (19)]. Since the magnitude of $s_{p}$ at $3 \mathrm{GHz}$ is large, the far field of the residual wave of $K_{x x}^{A}$ will be dominated by the lower term of (22) for all values of $\rho$, and bearing in mind that the continuous spectrum dominates the far field of $K_{x x}^{A}$ at $3 \mathrm{GHz}$ (see the comments of Fig. 5), the behavior of this far field must be of the type $\rho^{-2}$. In fact, Fig. 7 clearly shows that this behavior is reached for $k_{0} \rho \gtrsim 5$ at $3 \mathrm{GHz}$.

At $f=3.95 \mathrm{GHz}, s_{p} / k_{0}$ is found to be very close to zero and is associated with a improper real $k_{p}$ pole that is located very close to the branch point $k_{0}\left(k_{p} / k_{0}=1.0035709\right)$. This situation is always encountered when the frequency is below and close to the cutoff frequency of a surface wave mode of the structure (and now the frequency is below and close to the cutoff frequency of the first TE mode of the grounded dielectric slab). Since $s_{p}$ is very small in this case, $s$ can no longer 


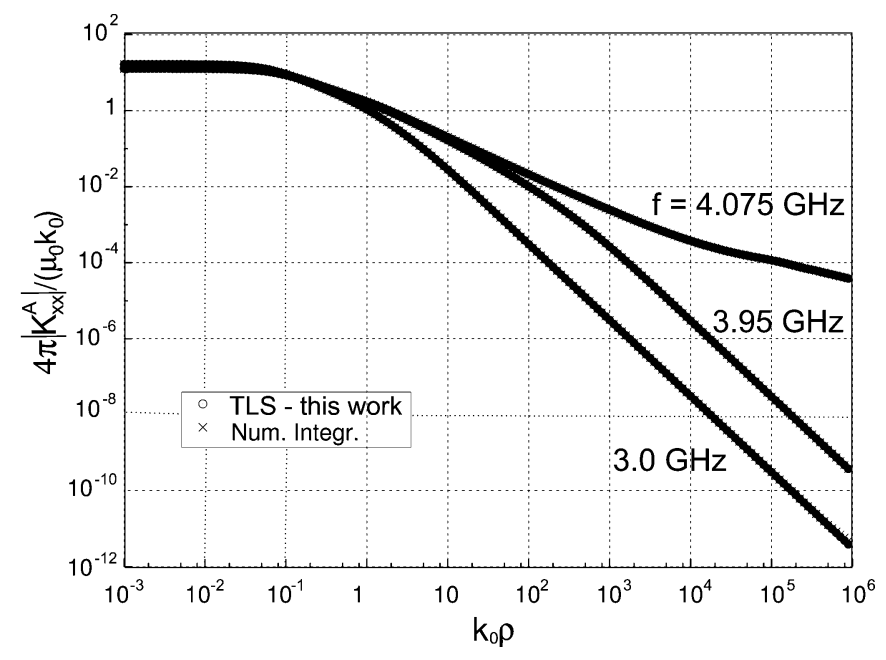

Fig. 7. Magnitude of the spatial-domain Green's function $K_{x x}^{A}$ at three different frequencies. Numerical integration results $(x)$ are compared with those obtained via (39) $(\odot)$. Parameters as in Fig. 5.

be neglected in the denominator of the approximation in (19) for small $s$. As a consequence, the behavior of $\tilde{K}_{x x}^{A,\left(k_{0}\right)}\left(s, z, z^{\prime}\right)$ at $3.95 \mathrm{GHz}$ in Fig. 6 is of the type $\sqrt{s}$ for $s / k_{0} \lesssim 0.005$ (as it happens at $3 \mathrm{GHz}$ ), but it suddenly transitions to a $1 / \sqrt{s}$ behavior for $s / k_{0} \gtrsim 0.005$ (which is not present at $3 \mathrm{GHz}$ ). This dual behavior of $\tilde{K}_{x x}^{A,\left(k_{0}\right)}\left(s, z, z^{\prime}\right)$ for small $s$ will always be present at those frequencies for which $s_{p}$ is close to zero (and, hence, $k_{p}$ is close to $k_{0}$ ). Since the improper pole $k_{p}$ obtained at $3.95 \mathrm{GHz}$ is not located in the shadowed region of Fig. 3, it does not contribute to the continuous spectrum of $K_{x x}^{A}$ and, therefore, it does not have to be explicitly incorporated in the approximation of (39) (in other words, in this case, $K_{x x}^{A,(\mathrm{cp})}=0$ ). This means that the residual wave dominates the far field of the continuous spectrum, and given that $3.95 \mathrm{GHz}$ is still below the cutoff frequency of the first TE mode, the residual wave will also dominate the far-field behavior of $K_{x x}^{A}$. Since $s_{p}$ is small at $3.95 \mathrm{GHz}$, the far-field behavior of both the residual wave and $K_{x x}^{A}$ will be a combination of the two behaviors shown in (22). This is clearly shown in Fig. 7 where the plot of $K_{x x}^{A}$ for $3.95 \mathrm{GHz}$ shows an intermediate zone (for $1 \lesssim k_{0} \rho \lesssim 500$ ) with a behavior of the type $\rho^{-1}$ that transitions to a behavior of the type $\rho^{-2}$ for $k_{0} \rho \gtrsim 500$ [in agreement with the overall behavior predicted by (22)]. When Watson's lemma is applied to the asymptotic evaluation of the integral in (16) [41], it is easy to show that the far-field behavior of the type $\rho^{-2}$ of $K_{x x}^{A}$ is related to the behavior of the type $\sqrt{s}$ of $\tilde{K}_{x x}^{A,\left(k_{0}\right)}\left(s, z, z^{\prime}\right)$ as $s \rightarrow 0$, and the far-field behavior of the type $\rho^{-1}$ of $K_{x x}^{A}$ is related to the behavior of the type $1 / \sqrt{s}$ of $\tilde{K}_{x x}^{A,\left(k_{0}\right)}\left(s, z, z^{\prime}\right)$ as $s \rightarrow 0$. Thus, the dual far-field behavior of $K_{x x}^{A}$ in Fig. 7 at $3.95 \mathrm{GHz}$ is a direct consequence of the dual behavior of $\tilde{K}_{x x}^{A,\left(k_{0}\right)}\left(s, z, z^{\prime}\right)$ for small $s$ in Fig. 6 at this frequency.

At a slightly higher frequency, $f=4.075 \mathrm{GHz}$, the TE mode is just above cutoff, and the pole $k_{p}$ is now a proper real pole that is located very close to the branch point $k_{0}\left(k_{p} / k_{0}=1.0000271\right)$. Due to it, $s_{p}=\left(k_{0}-k_{p}\right) / \mathrm{j}$ is also very close to zero and, as it happens at $3.95 \mathrm{GHz}$, the function $\tilde{K}_{x x}^{A,\left(k_{0}\right)}\left(s, z, z^{\prime}\right)$ of Fig. 6 experiences at $4.075 \mathrm{GHz}$ a transition from a behavior of the type $\sqrt{s}$ to a behavior of the type $1 / \sqrt{s}$ when $s / k_{0} \sim 5 \times 10^{-5}$. Since the pole $k_{p}$ is now located in the upper Riemann sheet, it belongs to the discrete spectrum of $K_{x x}^{A}$ and, therefore, it has to be explicitly incorporated in the approximation of (39) (i.e., $K_{x x}^{A,(\mathrm{cp})} \neq 0$ at $4.075 \mathrm{GHz}$ ). In principle, the far-field behavior of $K_{x x}^{A}$ at $4.075 \mathrm{GHz}$ should be dominated by the TE surface wave mode above cutoff. However, the effect of the continuous spectrum on the far field of $K_{x x}^{A}$ is strong, mostly because of the $\rho^{-1}$ term contributed by the residual wave to the far field of $K_{x x}^{A}$ as a consequence of the small value of $s_{p}$ (and ultimately, as a consequence of the behavior of the type $1 / \sqrt{s}$ shown by $\tilde{K}_{x x}^{A,\left(k_{0}\right)}\left(s, z, z^{\prime}\right)$ in Fig. 6 for small $s$ ). This is shown in Fig. 7 where the values of $K_{x x}^{A}$ for $4.075 \mathrm{GHz}$ show a behavior of the type $\rho^{-1}$ for a wide range of distances $\left(1 \lesssim k_{0} \rho \lesssim 5 \times 10^{4}\right)$, and then transition to a far-field behavior of the type $\rho^{-1 / 2}$, which is typical of surface waves.

Next, the lossy structure previously analyzed in [20, Fig. 4(b)] is reexamined in Fig. 8. Fig. 8(a) shows that the implementation of the method of total least squares via (38) makes it possible to obtain a good approximation of the spectral-domain scalar potential Green's function when losses are present. The spatial-domain behavior of $K_{\phi}$ is depicted in Fig. 8(b). As it happens with Fig. 5(b), the results obtained for $K_{\phi}$ with the approach of Section II agree very well with those obtained via numerical integration both in the near and far fields (the maximum relative error between these two set of results is below $1 \%$ along all the six decades considered). However, the results obtained with the approach of [7] slightly deviate from the numerical integration results in the interval $10 \lesssim k_{0} \rho \lesssim 1000$, and strongly differ from the numerical integration results when $k_{0} \rho \gtrsim 1000$. In Fig. 8(b), the far field of $K_{\phi}$ is dominated by the continuous spectrum since the presence of losses causes the exponential decay of the surface waves that compose the discrete spectrum of $K_{\phi}$. This last exponential decay can be observed in the interval $10^{2} \lesssim k_{\rho} \rho \lesssim 10^{3}$, and it changes to a behavior of the type $\rho^{-2}$ after a small transition region [this behavior of the type $\rho^{-2}$ is the contribution of the continuous spectrum as predicted by (22)]. The results of Fig. 8(b) obtained via (39) (circles) have also been compared with the results reported in [20, Fig. 4(b)], and good agreement has been found.

Finally, results for a four-layer microstrip structure containing a lossy silicon layer $(\sigma=10 \mathrm{~S} / \mathrm{m})$ are plotted in Fig. 9(a) and (b) in order to show the capabilities of the present method when dealing with lossy multilayered media both at low and high frequencies. A similar structure has previously been analyzed in the case where all the layers are lossless [7], [42]. The element of the vector potential Green's dyad that is studied in Fig. 9 is $K_{z x}^{A}$, which makes it possible to validate our approach when Hankel transforms of order 1 are involved. The low-frequency case is first analyzed in Fig. 9(a). In this situation, two different problems have to be faced, which are: 1) the low-frequency breakdown of any general full-wave electromagnetic approximation caused by the strong relevance of the quasi-static contribution in the near field [23] and 2) the presence of a very lossy substrate (at this low frequency, the silicon layer presents a very high value of the imaginary part 


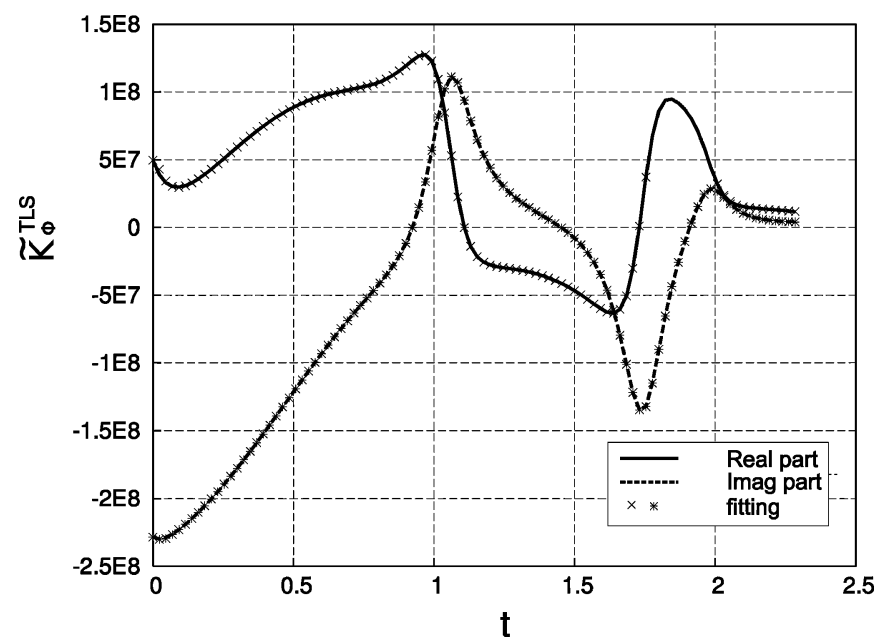

(a)

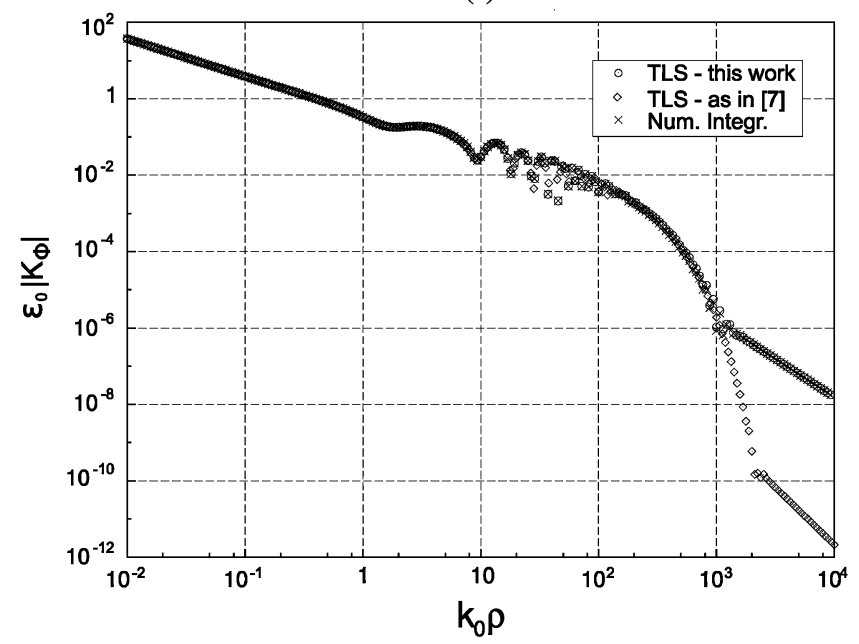

(b)

Fig. 8. (a) Real and imaginary parts of the spectral-domain functions $\tilde{K}_{\phi}^{\mathrm{TLS}(\mathrm{e})}$ (solid and dashed lines) and $\tilde{K}_{\phi}^{\mathrm{TLS}(\mathrm{pr})}(\times, *)$ along path $C$ of [7, Fig. 1]. Maximum relative error: $\leq 0.015$. (b) Magnitude of the spatial-domain Green's function $K_{\phi}$. Numerical integration results $(\times)$ are compared with those obtained via [7, eq. (13)] $(\diamond)$ and with those obtained via (39) $(\odot)$. Maximum relative error between $\times$ and $\odot$ is smaller than 0.01 . Parameters of the total least squares (TLS): $A=0.1, T_{0}=2.2, M=12$, and $N=27$. Parameters of the structure: $f=10 \mathrm{GHz}, z^{\prime}=z=0, N_{\mathrm{la}}=1 ; \varepsilon_{r 1}=4.4, \tan \delta_{1}=$ $0.02, h_{1}=10 \mathrm{~mm}$.

of the permittivity). After extracting out five static images via Prony's method, both problems are appropriately coped with by the present approach, and this is clearly shown in Fig. 9(a) where the relative error between our approach and numerical integration is always below $1 \%$. An additional complication that appears at this low-frequency concerns the presence of a proper pole $k_{p} / k_{0}=1.00004$ very close to the branch point. This situation has been well accounted for by the strategy followed in (36) and (37). The high-frequency case shown in Fig. 9(b) presents a completely different scenario. In this situation, the quasi-static fields are hardly relevant, and the far-field contribution is now the most significant part. Fortunately the method of Section II can handle this situation satisfactorily as it is demonstrated by the low values of the relative errors along all the range of $k_{0} \rho$ shown in Fig. 9(b). It should also be noted that the results obtained following [7, eq. (14)] would not provide

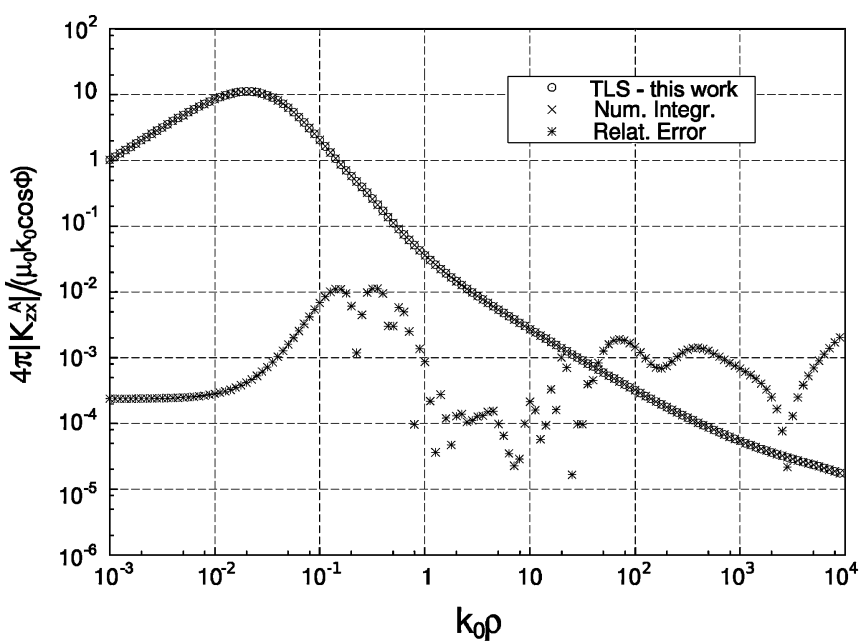

(a)

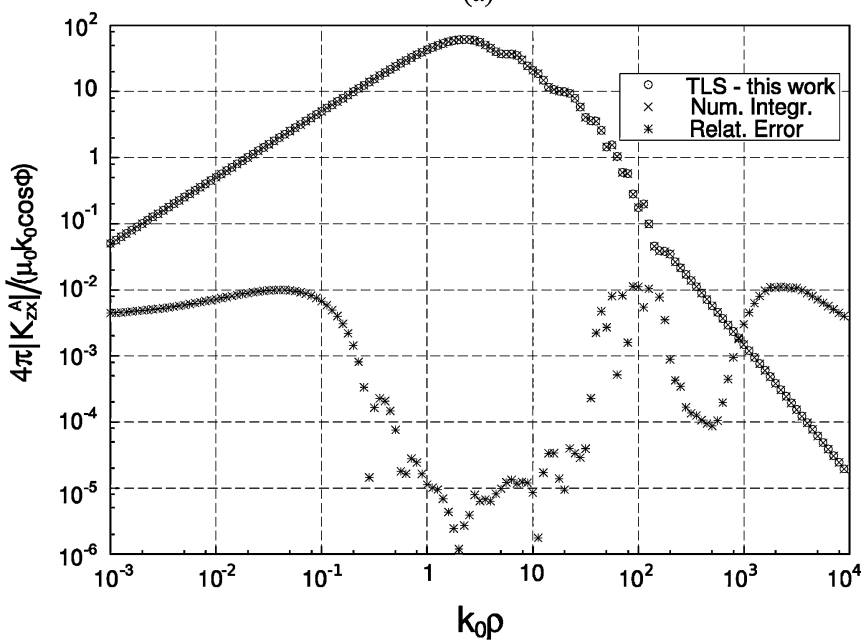

(b)

Fig. 9. Magnitude of the spatial-domain Green's function $K_{z x}^{A}$. (a) Freq $=$ $1 \mathrm{GHz}$. (b) Freq $=60 \mathrm{GHz}$. Numerical integration results $(+)$ are compared with those obtained via $(39)(\odot)$. The relative error $(x)$ between these two sets of values is also shown. Parameters of the total least squares (TLS): $A=$ $0.1, T_{0}=3.7, M=13$, and $N=29$. Parameters of the structure: $z^{\prime}=-1.4$ $\mathrm{mm}, z=-0.4 \mathrm{~mm}, N_{\mathrm{la}}=4 ; \varepsilon_{r 1}=2.1, \tan \delta_{1}=0, h_{1}=0.7 \mathrm{~mm}$ $\varepsilon_{r 2}=11.9, \tan \delta_{2}=\sigma /\left(\omega \varepsilon_{0} \varepsilon_{r 2}\right), \sigma=10 \mathrm{~S} / \mathrm{m}, h_{2}=0.3 \mathrm{~mm} ; \varepsilon_{r 3}=$ $9.8, \tan \delta_{3}=0, h_{3}=0.5 \mathrm{~mm} ; \varepsilon_{r 4}=8.6, \tan \delta_{4}=0, h_{4}=0.3 \mathrm{~mm}$.

accurate results in the far-field region because of its inability to account for far-field behaviors of the type $\rho^{-2}$, which are typical of lossy structures [20].

\section{CONCLUSION}

The authors have presented a novel implementation of the rational function fitting method, which uses the method of total least squares previously reported in [7] for obtaining closedform expressions of the multilayered media Green's functions introduced in [36, Form. C] for mixed potential integral equations. In this novel implementation, special attention has been paid to obtaining representations of the Green's functions that are accurate in the far field when this far field is not only dominated by surface waves, but also when it is dominated by the continuous spectrum. Thus, the new approach leads to closedform Green's functions that are accurate in the far field in the cases where lossy materials are involved, in the cases where the 
spectral-domain Green's functions have no proper poles, and in the two former cases when the spectral-domain Green's functions have one proper or improper pole that is very close to the branch point. The numerical results obtained with the new approach have proven to be accurate both in the near and far fields in situations when the far field is dominated either by the continuous spectrum or by surface waves. This sets our approach at the same level of generality as, for instance, that reported in [20]. However, it should be noticed that our method does not involve any numerical integration and that the highest dimension of the matrices involved in the numerical computation of the total least squares method has never gone beyond 30 (that of the matrices involved in Prony's method has never gone beyond 5). Thus, despite the generality and robustness of the method presented for the determination of spatial-domain Green's functions, the overall computation effort of the method is very competitive when compared with other methods.

\section{APPENDIX I}

In the specific case of a grounded dielectric slab of height $h$ and permittivity $\varepsilon=\varepsilon_{0} \varepsilon_{r}(1-\mathrm{j} \tan \delta)$ (i.e., the layered medium of Fig. 1 with $N_{\mathrm{la}}=1, \varepsilon_{1}=\varepsilon$ and $h_{1}=h$ ), when the field and source points are located on the air-dielectric interface $(z \equiv$ $z^{\prime}=0$ in Fig. 1), the coefficients $M_{p}$ and $s_{p}$ of (19) can be easily obtained in closed form for the different spectral-domain Green's functions involved in [36, Form. C]. The expressions of these coefficients are given by the following.

- $\tilde{K}_{x x}^{A}$

$$
\begin{gathered}
M_{p}=\mu_{0} \sqrt{\frac{2 \mathrm{j}}{k_{0}}} \\
s_{p}=\frac{\mathrm{j} C_{x x}^{2}}{2 k_{0}}
\end{gathered}
$$

where

$$
C_{x x}=\left(k_{0} \sqrt{\left(\varepsilon / \varepsilon_{0}\right)-1}\right) \operatorname{cotan}\left(k_{0} h \sqrt{\left(\varepsilon / \varepsilon_{0}\right)-1}\right) .
$$

- $\tilde{K}_{\phi}$

$$
\begin{aligned}
M_{p} & =\frac{1}{\epsilon_{0}} \sqrt{\frac{2 \mathrm{j}}{k_{0}}} \frac{B_{x x}\left[B_{x x}+C_{x x}\left(\left(\varepsilon / \varepsilon_{0}\right)-1\right)\right]}{B_{x x}^{2}+C_{x x}^{2}\left(\varepsilon / \varepsilon_{0}\right)^{2}} \\
s_{p} & =\frac{\mathrm{j}}{2 k_{0}} \frac{B_{x x}^{2} C_{x x}^{2}}{B_{x x}^{2}+C_{x x}^{2}\left(\varepsilon / \varepsilon_{0}\right)^{2}}
\end{aligned}
$$

where

$$
B_{x x}=-\left(k_{0} \sqrt{\left(\varepsilon / \varepsilon_{0}\right)-1}\right) \tan \left(k_{0} h \sqrt{\left(\varepsilon / \varepsilon_{0}\right)-1}\right) .
$$

- $\tilde{K}_{z z}^{A}$

$$
\begin{aligned}
M_{p} & =\mu_{o} \sqrt{\frac{2 \mathrm{j}}{k_{0}}} \frac{C_{x x}\left[C_{x x}\left(\varepsilon / \varepsilon_{0}\right)^{2}-B_{x x}\left(\left(\varepsilon / \varepsilon_{0}\right)-1\right)\right]}{B_{x x}^{2}+C_{x x}^{2}\left(\varepsilon / \varepsilon_{0}\right)^{2}} \\
s_{p} & =\frac{\mathrm{j}}{2 k_{0}} \frac{B_{x x}^{2} C_{x x}^{2}}{B_{x x}^{2}+C_{x x}^{2}\left(\varepsilon / \varepsilon_{0}\right)^{2}} .
\end{aligned}
$$

$$
\begin{aligned}
& \cdot \tilde{K}_{z x}^{A} / \mathrm{j} k_{x}=\tilde{K}_{z y}^{A} / \mathrm{j} k_{y}=-\tilde{K}_{x z}^{A} / \mathrm{j} k_{x}=-\tilde{K}_{y z}^{A} / \mathrm{j} k_{y} \\
& M_{p}=-\mu_{o} \sqrt{\frac{2 \mathrm{j}}{k_{0}}} \frac{\left(\left(\varepsilon / \varepsilon_{0}\right)-1\right)\left(B_{x x}+C_{x x}\left(\varepsilon / \varepsilon_{0}\right)\right)}{B_{x x}^{2}+C_{x x}^{2}\left(\varepsilon / \varepsilon_{0}\right)^{2}} \\
& s_{p}=\frac{\mathrm{j}}{2 k_{0}} \frac{B_{x x}^{2} C_{x x}^{2}}{B_{x x}^{2}+C_{x x}^{2}\left(\varepsilon / \varepsilon_{0}\right)^{2}} .
\end{aligned}
$$

\section{APPENDIX II}

$$
\begin{aligned}
& \tilde{G}_{1}^{\mathrm{RW}, \mathrm{ff}}\left(k_{\rho}, z, z^{\prime}\right) \\
& =\frac{2 \pi}{k_{\rho}} \int_{0}^{\infty} G_{1}^{\mathrm{RW}, \mathrm{ff}}\left(\rho, z, z^{\prime}\right) J_{1}\left(k_{\rho} \rho\right) \rho \mathrm{d} \rho \\
& =M_{p} k_{0} \sqrt{\frac{\mathrm{j} k_{0}}{2}}\left\{\sum _ { i = 1 } ^ { N _ { \mathrm { py } } } A _ { i } ^ { \mathrm { py } } \left[F\left(k_{\rho}, \mathrm{j} k_{0}+b_{i}^{\mathrm{py}}\left|s_{p}\right|\right)\right.\right. \\
& -3 F\left(k_{\rho},(\mathrm{j}+1) k_{0}+b_{i}^{\mathrm{py}}\left|s_{p}\right|\right) \\
& +3 F\left(k_{\rho},(\mathrm{j}+2) k_{0}+b_{i}^{\mathrm{py}}\left|s_{p}\right|\right) \\
& \left.-F\left(k_{\rho},(\mathrm{j}+3) k_{0}+b_{i}^{\mathrm{py}}\left|s_{p}\right|\right)\right] \\
& -\frac{1}{2 s_{p}}\left[D\left(k_{\rho}, \mathrm{j} k_{0}\right)-3 D\left(k_{\rho},(\mathrm{j}+1) k_{0}\right)\right. \\
& +3 D\left(k_{\rho},(\mathrm{j}+2) k_{0}\right) \\
& -D\left(k_{\rho},(\mathrm{j}+3) k_{0}\right) \\
& -D\left(k_{\rho}, \mathrm{j} k_{0}+\left|s_{p}\right|\right) \\
& +3 D\left(k_{\rho},(\mathrm{j}+1) k_{0}+\left|s_{p}\right|\right) \\
& -3 D\left(k_{\rho},(\mathrm{j}+2) k_{0}+\left|s_{p}\right|\right) \\
& \left.\left.+D\left(k_{\rho},(\mathrm{j}+3) k_{0}+\left|s_{p}\right|\right)\right]\right\}
\end{aligned}
$$

with

$$
\begin{aligned}
& D\left(k_{\rho}, x\right)=\left[x+\sqrt{k_{\rho}^{2}+x^{2}}\right]^{-1} \\
& F\left(k_{\rho}, x\right)=\left[\sqrt{k_{\rho}^{2}+x^{2}}\right]^{-1} D\left(k_{\rho}, x\right) .
\end{aligned}
$$

\section{ACKNOWLEDGMENT}

In the middle of a set of productive e-mail discussions between Prof. K. A. Michalski, Texas A\&M University, College Station, and the authors, Prof. K. A. Michalski addressed that both the discrete complex image method and the rational function fitting method failed to provide accurate far-field values of the Green's functions in lossy layered media. This claim prompted the authors to solve the far-field problem in the case of the rational function fitting method, and it finally gave rise to this paper. The authors are indebted to Prof. K. A. Michalski for being the starting point of this paper.

\section{REFERENCES}

[1] J. R. Mosig, "Arbitrarily shaped microstrip structures and their analysis with a mixed potential integral equation," IEEE Trans. Microw. Theory Tech., vol. 36, no. 2, pp. 314-323, Feb. 1988. 
[2] C. Chen, M. J. Tsai, and N. G. Alexopoulos, "Optimization of aperture transitions for multiport microstrip circuits," IEEE Trans. Microw. Theory Tech., vol. 44, no. 12, pp. 2457-2465, Dec. 1996.

[3] R. C. Hall and J. R. Mosig, "The analysis of arbitrarily shaped aperturecoupled patch antennas via a mixed-potential integral equation," IEEE Trans. Antennas Propag., vol. 44, no. 5, pp. 608-614, May 1996.

[4] C. Chen, W. E. McKinzie III, and N. G. Alexopoulos, "Stripline-fed arbitrarily shaped printed-aperture antennas," IEEE Trans. Antennas Propag., vol. 45, no. 7, pp. 1186-1198, Jul. 1997.

[5] J. R. Mosig, , T. Itoh, Ed., "Integral equation technique," in Numerical Techniques for Microwave and Millimeter-Wave Passive Structures. New York: Wiley, 1989, pp. 133-213.

[6] K. A. Michalski and J. R. Mosig, "Multilayered media Green's functions in integral equation formulations," IEEE Trans. Antennas Propag., vol. 45, no. 3, pp. 508-519, Mar. 1997.

[7] R. R. Boix, F. Mesa, and F. Medina, "Application of total least squares to the derivation of closed-form Green's functions for planar layered media," IEEE Trans. Microw. Theory Tech., vol. 55, no. 2, pp. 268-280, Feb. 2007.

[8] Y. L. Chow, J. J. Yang, D. G. Fang, and G. E. Howard, "A closed-form spatial Green's function for the thick microstrip substrate," IEEE Trans. Microw. Theory Tech., vol. 39, no. 3, pp. 588-592, Mar. 1991.

[9] M. I. Aksun, "A robust approach for the derivation of closed-form Green's functions," IEEE Trans. Microw. Theory Tech., vol. 44, no. 5, pp. 651-658, May 1996.

[10] N. V. Shuley, R. R. Boix, F. Medina, and M. Horno, "On the fast approximation of Green's functions in MPIE formulations for planar-layered media," IEEE Trans. Microw. Theory Tech., vol. 50, no. 9, pp. 2185-2192, Sep. 2002.

[11] M. I. Aksun and G. Dural, "Clarification of issues on the closed-form Green's functions in stratified media," IEEE Trans. Antennas Propag., vol. 53, no. 11, pp. 3644-3653, Nov. 2005.

[12] M. Yuan, T. K. Sarkar, and M. Salazar-Palma, "A direct discrete complex image method from the closed-form Green's functions in multilayered media," IEEE Trans. Microw. Theory Tech., vol. 54, no. 3, pp. 1025-1032, Mar. 2006

[13] S. A. Teo, S. T. Chew, and M. S. Leong, "Error analysis of the discrete complex image method and pole extraction," IEEE Trans. Microw. Theory Tech., vol. 51, no. 2, pp. 406-413, Feb. 2003.

[14] M. Zhang, L.-W. Li, and Y.-F. Tian, "An efficient approach for extracting poles of Green's functions in general multilayered media," IEEE Trans. Antennas Propag., vol. 53, no. 1, pp. 269-273, Jan. 2008.

[15] V. N. Kourkoulos and A. C. Cangellaris, "Accurate approximation of Green's functions in planar stratified media in terms of a finite sum of spherical and cylindrical waves," IEEE Trans. Antennas Propag., vol. 54, no. 5, pp. 1568-1576, May 2006.

[16] V. I. Okhmatovski and A. C. Cangellaris, "A new technique for the derivation of closed-form electromagnetic Green's functions for unbounded planar layered media," IEEE Trans. Antennas Propag., vol. 50, no. 7, pp. 1005-1016, Jul. 2002.

[17] A. G. Polimeridis, T. V. Yioultsis, and T. D. Tsiboukis, "Fast numerical computation of Green's functions for unbounded planar stratified media with a finite-difference technique and Gaussian spectral rules," IEEE Trans. Microw. Theory Tech., vol. 55, no. 1, pp. 100-107, Jan. 2007.

[18] A. G. Polimeridis, T. V. Yioultsis, and T. D. Tsiboukis, "A robust method for the computation of Green's functions in stratified media," IEEE Trans. Antennas Propag., vol. 55, no. 7, pp. 1963-1969, Jul. 2007.

[19] L. B. Felsen and N. Marcuvitz, Radiation and Scattering of Waves. New York: Prentice-Hall, 1973.

[20] J. R. Mosig and A. A. Álvarez-Melcón, "Green's functions in lossy layered media: Integration along the imaginary axis and asymptotic behavior," IEEE Trans. Antennas Propag., vol. 51, no. 12, pp. 3200-3208, Dec. 2003.

[21] S. V. Huffel and J. Vandevalle, "The total least squares problem: computational aspects and analysis," in Frontiers in Applied Mathematics. Philadelphia, PA: SIAM, 1991, vol. 9.

[22] J. Rahman and T. K. Sarkar, "Deconvolution and total least squares in finding the impulse response of an electromagnetic system from measured data," IEEE Trans. Antennas Propag., vol. 43, no. 4, pp. 416-421, Apr. 1995.

[23] F. Ling, V. Okhmatovski, B. Song, and A. Dengi, "Systematic extraction of static images from layered media Green's function for accurate DCIM implementation," IEEE Antennas Wireless Propag. Lett., vol. 6, pp. 215-218, 2007.
[24] R. W. Hamming, Numerical Methods for Scientists and Engineers. New York: Dover, 1973, pp. 620-622.

[25] Y. L. Chow, J. J. Yang, and G. E. Howard, "Complex images for electrostatic field computation in multilayered media," IEEE Trans. Microw. Theory Tech., vol. 39, no. 7, pp. 1120-1125, Jul. 1991.

[26] P. R. Haddad and D. M. Pozar, "Anomalous mutual coupling between microstrip antennas," IEEE Trans. Antennas Propag., vol. 42, no. 11, pp. 1545-1549, Nov. 1994.

[27] S.-T. Peng and A. A. Oliner, "Guidance and leakage properties of a class of open waveguides: Part I-Mathematical formulations," IEEE Trans. Microw. Theory Tech., vol. MTT-29, no. 9, pp. 843-855, Sep. 1981.

[28] T. Rozzi and P. D. Sewell, "The continuous spectrum of open waveguides of non-separable cross section," IEEE Trans. Antennas Propag., vol. 40, no. 11, pp. 1283-1291, Nov. 1992.

[29] R. Shavit, "Dielectric cover effect on rectangular microstrip antenna array," IEEE Trans. Antennas Propag., vol. 42, no. 8, pp. 1180-1184, Aug. 1994.

[30] M. Marin, S. Barkeshli, and P. H. Pathak, "Efficient analysis of planar microstrip geometries using a closed-form asymptotic representation of the grounded dielectric slab Green's function," IEEE Trans. Microw. Theory Tech., vol. 37, no. 4, pp. 669-679, Apr. 1989.

[31] F. J. Demuynck, G. A. E. Vandenbosch, and A. R. Van de Capelle, "The expansion wave concept-Part I: Efficient calculation of spatial Green's functions in a stratified dielectric medium," IEEE Trans. Antennas Propag., vol. 46, no. 3, pp. 397-406, Mar. 1998.

[32] M. Marin, S. Barkeshli, and P. H. Pathak, "On the location of proper and improper surface wave poles for the grounded dielectric slab," IEEE Trans. Antennas Propag., vol. 38, no. 4, pp. 570-573, Apr. 1990.

[33] C. G. Hsu, R. F. Harrington, J. R. Mautz, and T. K. Sarkar, "On the location of leaky wave poles for a grounded dielectric slab," IEEE Trans. Microw. Theory Tech., vol. 39, no. 2, pp. 346-349, Feb. 1991.

[34] F. Mesa and M. Horno, "Computation of proper and improper modes in multilayered bianisotropic waveguides," IEEE Trans. Microw. Theory Tech., vol. 43, no. 1, pp. 233-235, Jan. 1995.

[35] P. Baccarelli, P. Burghignoli, F. Frezza, A. Galli, G. Lovat, and D. R. Jackson, "Uniform analytical representation of the continuous spectrum excited by dipole sources in a multilayer dielectric structure through weighted cylindrical leaky waves," IEEE Trans. Antennas Propag., vol. 52, no. 3, pp. 653-665, Mar. 2004.

[36] K. A. Michalski and D. Zheng, "Electromagnetic scattering and radiation by surfaces of arbitrary shape in layered media, part I: Theory," IEEE Trans. Antennas Propag., vol. 38, no. 3, pp. 335-344, Mar. 1990.

[37] D. R. Jackson, F. Mesa, M. J. Freire, D. P. Nyquist, and C. Di Nallo, "An excitation theory for bound modes, leaky modes, and residualwave currents on stripline structures," Radio Sci., vol. 35, no. 2, pp. 495-510, Mar.-Apr. 2000.

[38] M. Abramowitz and I. Stegun, Handbook of Mathematical Functions, 9th ed. New York: Dover, 1970.

[39] P. Baccarelli, P. Burghignoli, F. Frezza, A. Galli, G. Lovat, and D. R Jackson, "Approximate analytical evaluation of the continuous spectrum in a substrate-superstrate dielectric waveguide," IEEE Trans. Microw. Theory Tech., vol. 50, no. 12, pp. 2690-2701, Dec. 2002.

[40] P. Baccarelli, P. Burghinogli, G. Lovat, S. Paulotto, F. Mesa, and D. R. Jackson, "Direct modal transition from space wave to surface wave leakage on microstrip lines," Radio Sci., vol. 40, 2005, RS6017.

[41] C. M. Bender and S. A. Orszag, Advanced Mathematical Methods for Scientists and Engineers. New York: McGraw-Hill, 1978.

[42] F. Ling and J. M. Jin, "Discrete complex image method for Green's functions of general multilayer media," IEEE Microw. Guided Wave Lett., vol. 10, no. 10, pp. 400-402, Oct. 2000. 


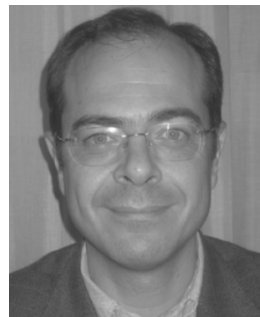

Rafael R. Boix (M'96) received the Licenciado and Doctor degrees in physics from the University of Seville, Seville, Spain, in 1985 and 1990, respectively.

Since 1986, he has been with the Department of Electronics and Electromagnetism, University of Seville, where, in 1994, he became an Associate Professor. His current research interests are focused on the numerical analysis of periodic electromagnetic structures with applications to the design of frequency-selective surfaces and electromagnetic bandgap passive circuits.

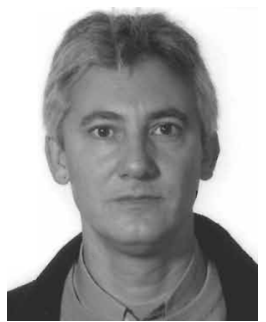

Francisco Medina (M'90-SM'01) received the Licenciado and Doctor degrees in physics from the University of Seville, Seville, Spain, in 1983 and 1987, respectively.

Since 1983, he has been with the Department of Electronics and Electromagnetism, University of Seville, where, in 1990, he became an Associate Professor. He is also currently the Head of the Microwaves Group with the Department of Electronics and Electromagnetism, University of Seville. His research interests include analytical and numerical methods for guiding, resonating and radiating structures, passive planar circuits, periodic structures, and the influence of anisotropic materials (including microwave ferrites) on such structures. He is also interested in artificial media modeling and design. 